\title{
Stereoselective Synthesis of Baylis-Hillman-Type Adducts via Allenolates Generated by Acyl Migration
}

\author{
Nagaraju Gudimalla, Roland Fröhlich ${ }^{\dagger}$ and Dieter Hoppe ${ }^{* *}$ \\ Organisch-Chemisches Institut, Westfälische Wilhelms-Universität Münster, Corrensstrasse 40, D-48149, \\ Germany, Fax+49-251-8336531, dhoppe@uni-muenster.de
}

\section{SUPPORTING INFORMATION}

\section{General Experimental Section}

All liquid aldehydes were distilled and stored under an argon atmosphere prior to use. The solid aldehydes were used without further purification. All reagents were dried and distilled prior to use.

Preparative flash column chromatography was performed under 1 bar argon pressure using silica gel grade 40-63 $\mu \mathrm{m}$ purchased from MERCK, Darmstadt. Solvents for column chromatography were distilled prior to their use. Thin layer chromatography was done using aluminium silica gel cards 60 F 254 purchased from MERCK, Darmstadt. The detection of the products was done under UV light at $254 \mathrm{~nm}$ as well as with staining agent ceric ammomium molybdate solution. The melting points were measured on a SMP3 melting point apparatus purchased from STUART SCIENTIFIC UK. Elemental analyses were performed at the microanalytical department of the Organisch-Chemisches Institut, WWU Münster, on a VARIO EL III purchased from ELEMENTAR ANALYSEN SYSTEME GmbH. IR measurements were performed on IR spectrometers IFS 28 purchased from BRUCKER and PE 298 purchased from PERKIN-ELMER \& Co GmbH, Überlingen. The mass spectral measurements were carried out by electron spray ionization method (ESI) on QUATTTRO LCZ (WATERS-MICROMASS, Manchester, UK) with a nanospray inlet at the Organisch-Chemisches Institut, WWU Münster. The ${ }^{1} \mathrm{H}$ and ${ }^{13} \mathrm{C}$ NMR spectra were measured on ARX300, AMX400, purchased from BRUKER ANALYTISCHE MESSTECHNIK, Karlsruhe, and 600 MHz UNITY PLUS purchased from VARIAN Associated, USA. $\mathrm{CDCl}_{3}$; TMS was used as internal standards for recording the NMR spectra.The signals are interpreted as s: singlet, br: broad, d: doublet, dd: doublet of doublet, t: triplet, m: multiplet, q: quartet. 


\section{General Experimental Procedures (GEP)}

\section{$\underline{\alpha-D e p r o t o n a t i o n ~ o f ~ a l l e n e s ~ a n d ~ s u b s e q u e n t ~ r e a c t i o n ~ w i t h ~ a c e t o n e ~}$}

\section{$\underline{\text { (GEP 1) }}$}

Allenyl carbamate 7a or $7 \mathbf{b}(1.0 \mathrm{mmol}, 1.0 \mathrm{eq})$ and TMEDA (1.2 mmol, $1.2 \mathrm{eq})$ were dissolved in diethyl ether $(5 \mathrm{~mL} / \mathrm{mmol})$ and cooled to $-78^{\circ} \mathrm{C} .1 .6 \mathrm{M} n$-BuLi in hexane $(1.2 \mathrm{mmol}, 1.2 \mathrm{eq})$ was added slowly to the solution and stirred for $20 \mathrm{~min}$. Acetone (3.0 mmol, $3.0 \mathrm{eq})$ was added slowly and the reaction mixture stirred for an additional $2 \mathrm{~h}$. The reaction mixture was quenched with $2 \mathrm{~N} \mathrm{HCl}(3.0 \mathrm{~mL} / \mathrm{mmol})$ at $-78^{\circ} \mathrm{C}$ and extracted with diethyl ether $(3 \times 30 \mathrm{~mL})$. The combined organic extracts were stirred over $\mathrm{NaHCO}_{3} / \mathrm{MgSO}_{4}$ and after filtration concentrated under vacuum. The crude material was purified by column chromatography (eluents: pentane/ $\mathrm{Et}_{2} \mathrm{O}=1: 1$ ) resulting in $\mathbf{8 a}$ or $\mathbf{8 b}$.

\section{Carbamoyl group migration and subsequent reaction with aldehydes at lower temperature to form (Z)-10a-10j (GEP 2)}

Allenyl carbamate $8 \mathbf{a}(1.0 \mathrm{mmol}, 1.0 \mathrm{eq})$ was dissolved in THF $(18.0 \mathrm{~mL} / \mathrm{mmol})$ and cooled to $-78{ }^{\circ} \mathrm{C} .1 .6 \mathrm{M} n$ - $\mathrm{BuLi}$ in hexane $(1.1 \mathrm{mmol}, 1.1 \mathrm{eq})$ was added with stirring. After $20 \mathrm{~min}$, the aldehyde $(1.2 \mathrm{mmol}, 1.2 \mathrm{eq})$ was added and the reaction mixture was warmed rapidly to $-40{ }^{\circ} \mathrm{C}$ and this temperature kept for $2.5 \mathrm{~h}$. The reaction mixture was quenched with water $(3.0 \mathrm{~mL} / \mathrm{mmol})$ at $-40{ }^{\circ} \mathrm{C}$ and extracted with diethyl ether $(3 \times 25 \mathrm{~mL})$. The combined organic extracts were dried over anhydrous $\mathrm{MgSO}_{4}$ and then concentrated under vacuum. The crude material was purified by column chromatography (eluents: pentane/ $\mathrm{Et}_{2} \mathrm{O}=5: 2$ ) resulting in $(Z)-\mathbf{1 0 a}-\mathbf{1 0 j}$.

Carbamoyl group migration and subsequent reaction with aldehydes at room temperature

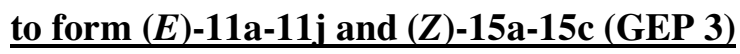

Allenyl carbamate $8 \mathbf{a}$ or $\mathbf{8 b}(1.0 \mathrm{mmol}, 1.0 \mathrm{eq})$ was dissolved in THF $(18.0 \mathrm{~mL} / \mathrm{mmol})$ and cooled to $-78{ }^{\circ} \mathrm{C} .1 .6 \mathrm{M} n$ BuLi in hexane (1.1 mmol, $1.1 \mathrm{eq})$ was added with stirring. After $20 \mathrm{~min}$, the reaction mixture was warmed rapidly to $-40{ }^{\circ} \mathrm{C}$ for $4 \mathrm{~h}$. The aldehyde (1.2 mmol, $\left.1.2 \mathrm{eq}\right)$ was added and the reaction mixture was stirred for an additional $1 \mathrm{~h}$ after which it was removed from the bath and stirred at room temperature for an additional $3-4 \mathrm{~h}$. The reaction mixture was quenched with water $(6.0 \mathrm{~mL} / \mathrm{mmol})$ and extracted with diethyl ether $(3 \times 25 \mathrm{~mL})$. The combined organic extracts were dried over anhydrous $\mathrm{MgSO}_{4}$ and then concentrated under vacuum. The crude material was purified by column chromatography (eluents: pentane/Et $\left.{ }_{2} \mathrm{O}=5: 2\right)$ furnishing $(E)-\mathbf{1 1 a}-\mathbf{1 1} \mathbf{j}$ or $(Z)-\mathbf{1 5 a} \mathbf{a}-15 \mathbf{c}$. 


\section{Synthesis of BAYLIS-HILLMAN-type adducts ${ }^{1}$}

\section{1-(1-Hydroxy-1-methylethyl)-4,4-dimethyl-1,2-pentadienyl) $N, N$-diisopropylcarbamate (8a)}

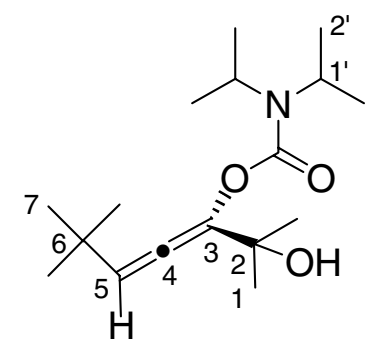

According to GEP 1, $500 \mathrm{mg}(2.09 \mathrm{mmol})$ of 7a resulted in $466 \mathrm{mg}(1.56 \mathrm{mmol}, 75 \%)$ 8a, as a colourless liquid; $\mathrm{R}_{\mathrm{f}}=$ $0.45\left(\mathrm{Et}_{2} \mathrm{O} /\right.$ pentane $\left.=1 / 1\right) ;{ }^{1} \mathrm{H}-\mathrm{NMR}(300 \mathrm{MHz}): \delta=1.09\left(\mathrm{~s}, 9 \mathrm{H}, 7-\mathrm{H}_{3}\right) ; 1.24\left(\mathrm{~d},{ }^{3} J_{2},{ }_{1}\right.$, $\left.=6.7 \mathrm{~Hz}, 12 \mathrm{H}, 2^{\prime}-\mathrm{H}_{3}\right) ; 1.36$ (2xs, $\left.6 \mathrm{H}, 1-\mathrm{H}_{3}\right) ; 3.65-3.95$ (br, m, $2 \mathrm{H}, 1$ '- $\left.\mathrm{H}_{1}\right) ; 4.08$ (s, $\left.1 \mathrm{H}, \mathrm{OH}\right) ; 5.74\left(\mathrm{~s}, 1 \mathrm{H}, 5-\mathrm{H}_{1}\right) ;{ }^{13} \mathrm{C}-\mathrm{NMR}(75 \mathrm{MHz}): \delta=21.04$, 21.22, 21.37 (C-2'); 28.53, 28.57 (C-1); 30.05 (C-7); 33.93 (C-6); 70.64 (C-2); 115.52 (C-5); 131.05 (C-3); 156.26 $(\mathrm{NC}=\mathrm{O}) ; 191.98(\mathrm{C}-4)$; IR (Film): $\tilde{\boldsymbol{V}}\left[\mathrm{cm}^{-1}\right]=3426(\mathrm{O}-\mathrm{H}) ; 2965,2904,2872(\mathrm{C}-\mathrm{H}) ; 1973(\mathrm{C}=\mathrm{C}=\mathrm{C}) ; 1683(\mathrm{NC}=\mathrm{O})$; ESI- MS: $\mathrm{m} / \mathrm{z}=298.4\left[\left(\mathrm{M}+\mathrm{H}^{+}\right)\right] ; 320.3\left[\left(\mathrm{M}+\mathrm{Na}^{+}\right)\right] ; \mathrm{C}_{17} \mathrm{H}_{31} \mathrm{NO}_{3}$ (297.23) Calcd. C 68.65, H 10.51, N 4.71; Found C 68.38, H 10.63, N 4.56.

\section{1-(1-Hydroxy-1-methylethyl)-1,2-butadienyl $N, N$-diisopropylcarbamate (8b)}

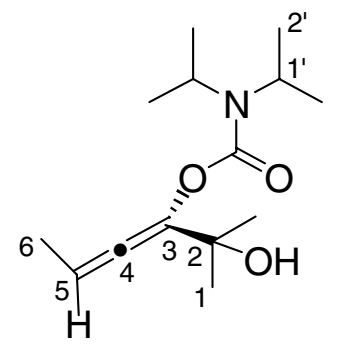

According to GEP 1, $1059 \mathrm{mg}(5.37 \mathrm{mmol})$ of $\mathbf{7 b}$ resulted in $932 \mathrm{mg}(3.65 \mathrm{mmol}, 68 \%) \mathbf{8 b}$, as a colourless liquid; $\mathrm{R}_{\mathrm{f}}=$ $0.5\left(\mathrm{Et}_{2} \mathrm{O} /\right.$ pentane $\left.=1 / 1\right) ;{ }^{1} \mathrm{H}-\mathrm{NMR}(300 \mathrm{MHz}): \delta=1.36\left(\mathrm{~d},{ }^{3} J_{2}, 1,=6.8 \mathrm{~Hz}, 12 \mathrm{H}, 2{ }^{\prime}-\mathrm{H}_{3}\right) ; 1.46\left(\mathrm{~d}, 6 \mathrm{H}, 1-\mathrm{H}_{3}\right) ; 1.90(\mathrm{~d}$, $\left.{ }^{3} J_{6,5}=7.1 \mathrm{~Hz}, 3 \mathrm{H}, 6-\mathrm{H}_{3}\right) ; 3.81(\mathrm{~s}, 1 \mathrm{H}, \mathrm{OH}) ; 3.88-4.32$ (br m, $2 \mathrm{H}, 1$ ' $\left.-\mathrm{H}_{1}\right) ; 5.84\left({ }^{3} J_{5,6}=7.1 \mathrm{~Hz}, 1 \mathrm{H}, 6-\mathrm{H}_{1}\right) ;{ }^{13} \mathrm{C}-\mathrm{NMR}$ (75 MHz): $\delta=15.66$ (C-6); 21.02, 21.72 (C-2'); 28.42, 28.45 (C-1); 47.17 (C-1'); 70.87 (C-2); 99.32 (C-5); 128.64 (C3); 155.60 (NC=O); 195.56 (C-4); IR (Film): $\tilde{V}\left[\mathrm{~cm}^{-1}\right]=3430$ (O-H); 2976, 2934, 2876 (C-H); 1974 (C=C=C); 1686 $(\mathrm{NC}=\mathrm{O})$; ESI- MS: $\mathrm{m} / \mathrm{z}=278.6\left[\left(\mathrm{M}+\mathrm{Na}^{+}\right)\right] ; \mathrm{C}_{14} \mathrm{H}_{25} \mathrm{NO}_{3}$ (255.18) Calcd. C 65.85, H 9.87, N 5.49; Found C 65.45, H 9.90, N 5.34 .

1 Numbering of NMR data does not follow IUPAC nomenclature. 


\section{(Z)-3-(1-Hydroxy-1-phenylmethyl)-1,1,5,5-tetramethyl-2-oxo-3-hexenyl}

\section{$N, N$-diisopropylcarbamate $((Z)-10 a)$}

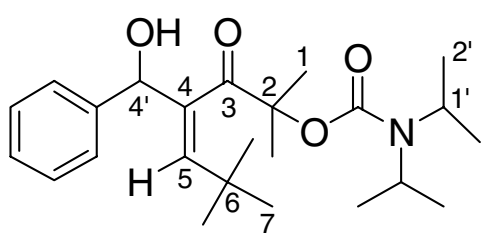

According to GEP 2, $320 \mathrm{mg}(1.07 \mathrm{mmol})$ of 8a resulted in $345 \mathrm{mg}(0.85 \mathrm{mmol}, 79 \%)(Z)-\mathbf{1 0 a}$, colourless solid; $\mathrm{R}_{\mathrm{f}}=$ $0.50\left(\mathrm{Et}_{2} \mathrm{O} /\right.$ pentane $\left.=2 / 5\right) ; \mathrm{m} . \mathrm{p} .=115{ }^{\circ} \mathrm{C}$ (from the melt); ${ }^{1} \mathrm{H}-\mathrm{NMR}(300 \mathrm{MHz}): \delta=1.00\left(\mathrm{~s}, 9 \mathrm{H}, 7-\mathrm{H}_{3}\right) ; 1.26(\mathrm{br} \mathrm{t}$, $\left.{ }^{3} J_{2}{ }^{\prime},{ }^{\prime}=6.7 \mathrm{~Hz}, 12 \mathrm{H}, 2^{\prime}-\mathrm{H}_{3}\right) ; 1.73,1.74\left(\mathrm{~s}, 6 \mathrm{H}, 1-\mathrm{H}_{3}\right) ; 3.09$ (d, $\left.{ }^{3} J_{\mathrm{OH}, 4^{\prime}}=4.8 \mathrm{~Hz}, 1 \mathrm{H}, \mathrm{OH}\right) ; 3.75-4.14$ (br m, $2 \mathrm{H}, 1$ '- $\left.-\mathrm{H}_{1}\right)$; $5.31\left(\mathrm{~d},{ }^{4} J_{5,4}=1.1 \mathrm{~Hz}, 1 \mathrm{H}, 5-\mathrm{H}_{1}\right) ; 5.66\left(\mathrm{dd},{ }^{3} J_{4}, \mathrm{OH}=4.8 \mathrm{~Hz},{ }^{4} J_{4}, 5=1.1 \mathrm{~Hz}, 1 \mathrm{H}, 4{ }^{\prime}-\mathrm{H}_{1}\right) ; 7.26-7.38(\mathrm{~m}, 5 \mathrm{H}, \mathrm{Ph}) ;{ }^{13} \mathrm{C}-$ NMR (75 MHz): $\delta=20.94$ (C-2’); 25.98, 26.72 (C-1); 30.19 (C-7); 33.48 (C-6); 46.20 (C-1'); 74.50 (C-4'); 85.88 (C2); 127.28, 127.67, 128.26 (CH-Ph); 140.83, 141.46 (C-4, C ipso-Ph); 143.63 (C-5); 153.46 (NC=O); 209.20 (C-3); IR $(\mathrm{KBr}): \tilde{V}\left[\mathrm{~cm}^{-1}\right]=3439(\mathrm{O}-\mathrm{H}) ; 2967(\mathrm{C}-\mathrm{H}) ; 1705(\mathrm{NC}=\mathrm{O}) ; 1679(\mathrm{C}=\mathrm{C}-\mathrm{C}=\mathrm{O}) ; 759,699(\mathrm{Ph}) ; \mathrm{ESI}-\mathrm{MS}: \mathrm{m} / \mathrm{z}=404.4$ $\left[\left(\mathrm{M}+\mathrm{H}^{+}\right)\right] ; 426.4\left[\left(\mathrm{M}+\mathrm{Na}^{+}\right)\right] ; \mathrm{C}_{24} \mathrm{H}_{37} \mathrm{NO}_{4}$ (403.27) Calcd. C 71.43, H 9.24, N 3.47; Found C 71.28, H 9.36, N 3.24.

\section{(E)-3-(1-Hydroxy-1-phenylmethyl)-1,1,5,5-tetramethyl-2-oxo-3-hexenyl}

\section{$N, N$ - diisopropylcarbamate $((E)-11 a)$}

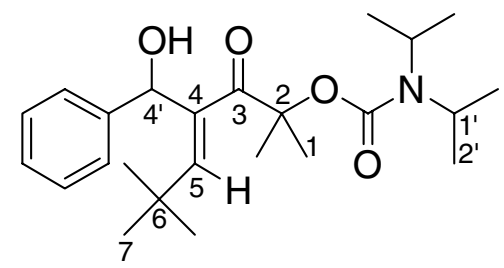

According to GEP 3, $475 \mathrm{mg}$ (1.59 mmol) of 8a resulted in $464 \mathrm{mg}(1.15 \mathrm{mmol}, 72 \%)(E)$-11a, colourless liquid which becomes a solid in the refrigerator; $\mathrm{R}_{\mathrm{f}}=0.53\left(\mathrm{Et}_{2} \mathrm{O} /\right.$ pentane $\left.=2 / 5\right) ;{ }^{1} \mathrm{H}-\mathrm{NMR}(300 \mathrm{MHz}): \delta=1.24\left(\mathrm{~s}, 9 \mathrm{H}, 7-\mathrm{H}_{3}\right)$;

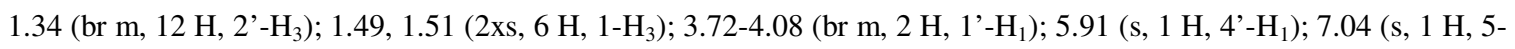
$\left.\mathrm{H}_{1}\right)$; 7.15-7.46 (m, $\left.5 \mathrm{H}, \mathrm{Ph}\right) ;{ }^{13} \mathrm{C}-\mathrm{NMR}(75 \mathrm{MHz}): \delta=21.03,22.24,22.41$ (C-2'); 26.37, $26.62(\mathrm{C}-1)$; 31.62 (C-7); 33.82 (C-6); 46.17, 47.15 (C-1'); 70.50 (C-4’); 83.93 (C-2); 126.04, 126.79, 128.19 (CH-Ph); 137.52 (C-4); 143.72 (C ipso- Ph); 152.12 (C-5); 154.32 (NC=O); 202.61 (C-3); IR (Film): $\tilde{v} \quad\left[\mathrm{~cm}^{-1}\right]=3449$ (O-H); 2960 (C-H); 1693 (NC=O); $1663(\mathrm{C}=\mathrm{C}-\mathrm{C}=\mathrm{O}) ; 771,733,698(\mathrm{Ph}) ; \mathrm{ESI}-\mathrm{MS}: \mathrm{m} / \mathrm{z}=404.6\left[\left(\mathrm{M}+\mathrm{H}^{+}\right)\right] ; 426.6\left[\left(\mathrm{M}+\mathrm{Na}^{+}\right)\right] ; \mathrm{C}_{24} \mathrm{H}_{37} \mathrm{NO}_{4}(403.27) \mathrm{Calcd}$. C 71.43, H 9.24, N 3.47; Found C 71.29, H 9.27; N 3.41. 


\section{(Z)-3-[1-(4-Bromophenyl)-1-hydroxymethyl]-1,1,5,5-tetramethyl-2-oxo-3-hexenyl $N, N$ -}

diisopropylcarbamate $((Z)-10 b)$

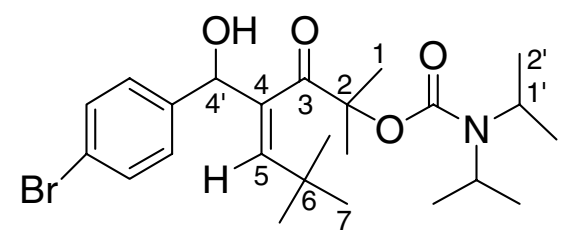

According to GEP 2, $122 \mathrm{mg}(0.41 \mathrm{mmol})$ of 8a resulted in $137 \mathrm{mg}(0.28 \mathrm{mmol}, 69 \%)(Z)-\mathbf{1 0 b}$, colourless solid; $\mathrm{R}_{\mathrm{f}}=$ $0.43\left(\mathrm{Et}_{2} \mathrm{O} /\right.$ pentane $\left.=2 / 5\right) ;$ m.p. $=131{ }^{\circ} \mathrm{C}($ from the melt $) ;{ }^{1} \mathrm{H}-\mathrm{NMR}(300 \mathrm{MHz}): \delta=1.00\left(\mathrm{~s}, 9 \mathrm{H}, 7-\mathrm{H}_{3}\right) ; 1.25\left(\mathrm{dd},{ }^{3} J_{2}, 1\right.$, $\left.=7.0 \mathrm{~Hz}, 12 \mathrm{H}, 2^{\prime}-\mathrm{H}_{3}\right) ; 1.70,1.73\left(\mathrm{~s}, 6 \mathrm{H}, 1-\mathrm{H}_{3}\right) ; 3.45\left(\mathrm{~d},{ }^{3} J_{\mathrm{OH}, 4}=4.2 \mathrm{~Hz}, 1 \mathrm{H}, \mathrm{OH}\right) ; 3.81-4.10\left(\mathrm{br} \mathrm{m}, 2 \mathrm{H}, 1^{\prime}-\mathrm{H}_{1}\right) ; 5.31$ $\left(\mathrm{d},{ }^{4} J_{5,4}=0.9 \mathrm{~Hz}, 1 \mathrm{H}, 5-\mathrm{H}_{1}\right) ; 5.58\left(\mathrm{~d},{ }^{3} J_{4}, \mathrm{OH}=3.2 \mathrm{~Hz}, 1 \mathrm{H}, 4{ }^{\prime}-\mathrm{H}_{1}\right) ; 7.19-7.29(\mathrm{~m}, 2 \mathrm{H}, \mathrm{Ph}) ; 7.41-7.49(\mathrm{~m}, 2 \mathrm{H}, \mathrm{Ph}) ;{ }^{13} \mathrm{C}-$ NMR (75 MHz): $\delta=21.42$ (C-2'); 26.55, 27.14 (C-1); 30.46 (C-7); 33.93 (C-6); 46.67 (C-1'); 74.52 (C-4'); 86.14 (C2); 121.82 (C ipso p-Br-Ph); 129.24, 131.67 (CH-Ph); 140.90, 141.19 (C-4, C ipso Ph); 144.27 (C-5); 153.96 (NC=O); 209.27 (C-3); IR (KBr): $\widetilde{v}\left[\mathrm{~cm}^{-1}\right]=3436(\mathrm{O}-\mathrm{H}) ; 2965(\mathrm{C}-\mathrm{H}) ; 1701(\mathrm{NC}=\mathrm{O}) ; 1679$ (C=C-C=O); ESI-MS: m/z = 482.4, $484.4\left[\left(\mathrm{M}+\mathrm{H}^{+}\right)\right] ; 504.3,506.3\left[\left(\mathrm{M}+\mathrm{Na}^{+}\right)\right] ; \mathrm{C}_{24} \mathrm{H}_{36} \mathrm{BrNO}_{4}$ (481.18) Calcd. C 59.75, H 7.52, N 2.90; Found C 59.65, H $7.44, \mathrm{~N} 2.88$.

\section{(E)-3-[1-(4-Bromophenyl)-1-hydroxymethyl]-1,1,5,5-tetramethyl-2-oxo-3-hexenyl $N, N$ -} diisopropylcarbamate $((E)-11 b)$<smiles>CC1CC(C)(C)C(C(C)(C)C)=C1C(=O)C(C)(C)OC(=O)N(C(C)C)C(C)C</smiles>

According to GEP 3, $150 \mathrm{mg}(0.50 \mathrm{mmol})$ of 8a resulted in $153 \mathrm{mg}(0.31 \mathrm{mmol}, 63 \%)(E)-\mathbf{1 1 b}$, colourless solid; $\mathrm{R}_{\mathrm{f}}=$ $0.53\left(\mathrm{Et}_{2} \mathrm{O} /\right.$ pentane $\left.=2 / 5\right)$; m.p. $=131{ }^{\circ} \mathrm{C}($ from the melt $) ;{ }^{1} \mathrm{H}-\mathrm{NMR}(300 \mathrm{MHz}): \delta=1.20-1.32\left(\right.$ br m, $\left.12 \mathrm{H}, 2^{\prime}-\mathrm{H}_{1}\right) ; 1.23$

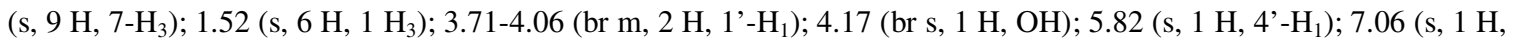
5-H $_{1}$; 7.30-7.44 (m, 4 H, Ar); ${ }^{13} \mathrm{C}-\mathrm{NMR}$ (75 MHz): $\delta=20.52,20.61,21.87,21.98$ (C-2'); 26.18 (C-1); 31.22 (C-7); 33.40 (C-6); 45.92, 46.80 (C-1'); 69.65 (C-4'); 83.59 (C-2); 120.38 (C ipso p-Br-Ph); 127.57, 130.88 (CH-Ph); 136.68 (C-4); 142.53 (C ipso Ph); $152.33(\mathrm{C}-5) ; 153.97(\mathrm{NC}=\mathrm{O}) ; 202.12(\mathrm{C}-3) ; \mathrm{IR}(\mathrm{KBr}): \tilde{v} \quad\left[\mathrm{~cm}^{-1}\right]=3516(\mathrm{O}-\mathrm{H}) ; 2974$, 2950, $2872(\mathrm{C}-\mathrm{H}) ; 1682(\mathrm{NC}=\mathrm{O}) ; 1646(\mathrm{C}=\mathrm{C}-\mathrm{C}=\mathrm{O})$; ESI-MS: $\mathrm{m} / \mathrm{z}=504.5,506.5\left[\left(\mathrm{M}+\mathrm{Na}^{+}\right)\right] ; \mathrm{C}_{24} \mathrm{H}_{36} \mathrm{BrNO}_{4}(481.18)$ Calcd. C 59.75, H 7.52, N 2.90; Found C 59.80, H 7.50, N 2.84. 


\section{(Z)-3-[1-Hydroxy-1-(4-nitrophenyl)methyl]-1,1,5,5-tetramethyl-2-oxo-3-hexenyl $N, N$ -} diisopropylcarbamate $((Z)-10 c)$

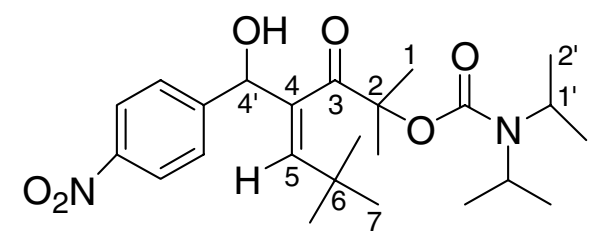

According to GEP 2, $106 \mathrm{mg}(0.35 \mathrm{mmol})$ of $\mathbf{8 a}$ resulted in $133 \mathrm{mg}(0.29 \mathrm{mmol}, 83 \%)(Z)$-10c, light yellow solid; $\mathrm{R}_{\mathrm{f}}=$ $0.33\left(\mathrm{Et}_{2} \mathrm{O} /\right.$ pentane $\left.=2 / 5\right) ; \mathrm{m} . \mathrm{p} .=146{ }^{\circ} \mathrm{C}$ (from the melt) $;{ }^{1} \mathrm{H}-\mathrm{NMR}(300 \mathrm{MHz}): \delta=0.99\left(\mathrm{~s}, 9 \mathrm{H}, 7-\mathrm{H}_{3}\right) ; 1.25\left(\mathrm{dd},{ }^{3} J_{2}, 1\right.$, = 6.9 Hz, $\left.12 \mathrm{H}, 2^{\prime}-\mathrm{H}_{3}\right) ; 1.70\left(\mathrm{~s}, 3 \mathrm{H}, 1-\mathrm{H}_{3}\right) ; 1.77$ (s, $\left.3 \mathrm{H}, 1-\mathrm{H}_{3}\right) ; 4.09$ (s, $1 \mathrm{H}, 1$ '- $\left.-\mathrm{H}_{1}\right) ; 4.35\left(\mathrm{~d},{ }^{3} J_{\mathrm{OH}, 4}\right.$, = 3.8 Hz, $\left.1 \mathrm{H}, \mathrm{OH}\right)$; $5.32\left(\mathrm{~s}, 1 \mathrm{H}, 5 \mathrm{H}_{1}\right) ; 5.65\left(\mathrm{~d},{ }^{3} \mathrm{~J}_{4}{ }^{\prime}, \mathrm{OH}=3.8 \mathrm{~Hz}, 1 \mathrm{H}, 4^{\prime}-\mathrm{H}_{1}\right) ; 7.53-7.61(\mathrm{~m}, 2 \mathrm{H}, \mathrm{Ph}), 8.13-8.22(\mathrm{~m}, 2 \mathrm{H}, \mathrm{Ph}) ;{ }^{13} \mathrm{C}-\mathrm{NMR}(75$ MHz): $\delta=21.39$ (C-2'); 26.94, 27.18 (C-1); 30.27 (C-7); 34.05 (C-6); 46.83 (C-1'); 74.91 (C-4'); 86.10 (C-2); 123.65 , 127.98 (CH-Ph); 140.35 (C-4); 144.91 (C-5); 147.57 (C ipso, Ph); 150.08 (C ipso p-NO $\left.\mathrm{NO}_{2}-\mathrm{Ph}\right) ; 154.25(\mathrm{NC}=\mathrm{O}) ; 209.13$ $(\mathrm{C}-3) ; \mathrm{IR}(\mathrm{KBr}): \tilde{v}\left[\mathrm{~cm}^{-1}\right]=3372(\mathrm{O}-\mathrm{H}) ; 2969(\mathrm{C}-\mathrm{H}) ; 1710(\mathrm{NC}=\mathrm{O}) ; 1676,1648(\mathrm{C}=\mathrm{C}-\mathrm{C}=\mathrm{O}) ; \mathrm{ESI}-\mathrm{MS}: \mathrm{m} / \mathrm{z}=449.5$ $\left[\left(\mathrm{M}+\mathrm{H}^{+}\right)\right] ; 471.4\left[\left(\mathrm{M}+\mathrm{Na}^{+}\right)\right]$; Exact mass $\mathrm{C}_{24} \mathrm{H}_{37} \mathrm{~N}_{2} \mathrm{O}_{6}$ Calcd. 449.27, Found 449.2646.

\section{(E)-3-[1-Hydroxy-1-(4-nitrophenyl)methyl]-1,1,5,5-tetramethyl-2-oxo-3-hexenyl $N, N$ -} diisopropylcarbamate $((E)-11 c)$

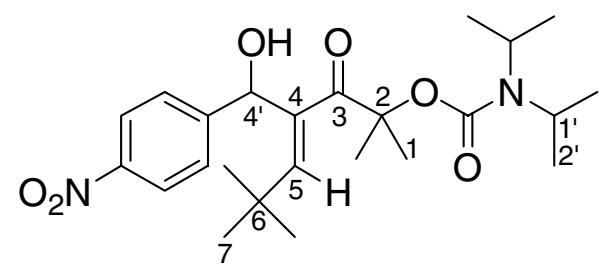

According to GEP 3, $150 \mathrm{mg}(0.50 \mathrm{mmol})$ of 8a resulted in $160 \mathrm{mg}(0.35 \mathrm{mmol}, 60 \%)(E)-\mathbf{1 1 c}$, colourless solid; $\mathrm{R}_{\mathrm{f}}=$ $0.43\left(\mathrm{Et}_{2} \mathrm{O} /\right.$ pentane $\left.=2 / 5\right) ; \mathrm{m} . \mathrm{p} .=131{ }^{\circ} \mathrm{C}$ (from the melt); ${ }^{1} \mathrm{H}-\mathrm{NMR}(300 \mathrm{MHz}): \delta=1.27\left(\mathrm{~s}, 9 \mathrm{H}, 7-\mathrm{H}_{3}\right) ; 1.20-1.34(\mathrm{~m}$, $\left.12 \mathrm{H}, 2^{\prime}-\mathrm{H}_{3}\right) ; 1.52$ (s, $\left.3 \mathrm{H}, 1-\mathrm{H}_{3}\right) ; 1.54$ (s, $\left.3 \mathrm{H}, 1-\mathrm{H}_{3}\right) ; 3.82-4.12$ (br m, $2 \mathrm{H}, 1$ '- $\left.-\mathrm{H}_{1}\right) ; 4.22$ (br s, $\left.1 \mathrm{H}, \mathrm{OH}\right) ; 5.91$ (s, $1 \mathrm{H}$, 4'- $\left.\mathrm{H}_{1}\right) ; 7.12$ (s, $\left.1 \mathrm{H}, 5-\mathrm{H}_{1}\right) ; 7.58-7.65$ (m, $\left.2 \mathrm{H}, \mathrm{Ph}\right) ; 8.15-8.21(\mathrm{~m}, 2 \mathrm{H}, \mathrm{Ph}) ;{ }^{13} \mathrm{C}-\mathrm{NMR}(75 \mathrm{MHz}): \delta=21.01,22.22$, 22.32 (C-2'); 26.51, 26.64 (C-1); 31.65 (C-7); 33.78 (C-6); 46.39, 47.26 (C-1'); 69.89 (C-4'); 83.94 (C-2); 123.53 , 126.69, 127.98 (CH-Ph); 136.82 (C-4); 147.03, 151.71 (C ipso, Ph); 153.23 (C-5); 154.49 (NC=O); 202.33 (C-3); IR $(\mathrm{KBr}): \tilde{V}\left[\mathrm{~cm}^{-1}\right]=3517(\mathrm{O}-\mathrm{H}) ; 2970,2935,2873(\mathrm{C}-\mathrm{H}) ; 1687(\mathrm{NC}=\mathrm{O}) ; 1650,1624(\mathrm{C}=\mathrm{C}-\mathrm{C}=\mathrm{O}) ; \mathrm{ESI}-\mathrm{MS}: \mathrm{m} / \mathrm{z}=$ $449.7\left[\left(\mathrm{M}+\mathrm{H}^{+}\right)\right] ; 471.6\left[\left(\mathrm{M}+\mathrm{Na}^{+}\right)\right] ; \mathrm{C}_{24} \mathrm{H}_{36} \mathrm{~N}_{2} \mathrm{O}_{6}$ (448.26) Calcd. C 64.26, H 8.09, N 6.25; Found C 64.25, H 7.92, N 5.98 . 


\section{(Z)-3-[1-Hydroxy-1-(4-methoxyphenyl)methyl]-1,1,5,5-tetramethyl-2-oxo-3-hexenyl $N, N$ -} diisopropylcarbamate $((Z)-10 d)$

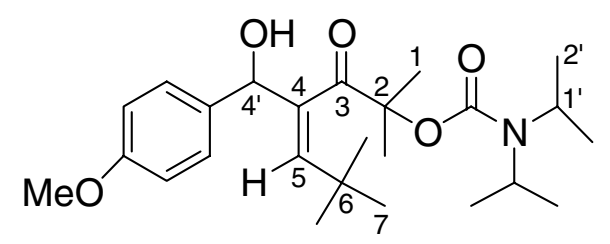

According to GEP 2, $117 \mathrm{mg}(0.39 \mathrm{mmol})$ of 8 a resulted in $155 \mathrm{mg}(0.36 \mathrm{mmol}, 91 \%)(Z)$-10d, white solid; $\mathrm{R}_{\mathrm{f}}=0.65$ $\left(\mathrm{Et}_{2} \mathrm{O} /\right.$ pentane $\left.=2 / 5\right) ;$ m.p. $=131{ }^{\circ} \mathrm{C}$ (from the melt); ${ }^{1} \mathrm{H}-\mathrm{NMR}(300 \mathrm{MHz}): \delta=1.02\left(\mathrm{~s}, 9 \mathrm{H}, 7-\mathrm{H}_{3}\right) ; 1.25\left(\mathrm{dd}^{3} J_{2^{\prime}, 1},=6.8\right.$ $\left.\mathrm{Hz}, 12 \mathrm{H}, 1^{\prime}-\mathrm{H}_{3}\right) ; 1.69$ (s, $\left.3 \mathrm{H}, 1-\mathrm{H}_{3}\right) ; 1.72$ (s, $\left.3 \mathrm{H}, 1-\mathrm{H}_{3}\right) ; 2.80\left(\mathrm{~d},{ }^{3} J_{\mathrm{OH}, 4^{\prime}}=4.8 \mathrm{~Hz}, 1 \mathrm{H}, \mathrm{OH}\right) ; 3.62-4.20$ (br m, 2 H, 1'$\left.\mathrm{H}_{1}\right) ; 3.82(\mathrm{~s}, 3 \mathrm{H}, \mathrm{OMe}) ; 5.39\left(\mathrm{~d},{ }^{4} J_{5,4^{\prime}}=1.3 \mathrm{~Hz}, 1 \mathrm{H}, 5-\mathrm{H}_{1}\right) ; 5.59\left(\mathrm{~d},{ }^{3} J_{4^{\prime}, \mathrm{OH}}=4.8 \mathrm{~Hz}, 1 \mathrm{H}, 4^{\prime}-\mathrm{H}_{1}\right) ; 6.82-6.91(\mathrm{~m}, 2 \mathrm{H}$, $\mathrm{Ph}$ ); 7.21-7.30 (m, $2 \mathrm{H}, \mathrm{Ph}) ;{ }^{13} \mathrm{C}-\mathrm{NMR}$ (75 MHz): $\delta=21.39$ (C-2'); 26.38, 26.89 (C-1); 30.66 (C-7); 33.77 (C-6); 46.53 (C-1'); $55.60\left(\mathrm{Ar}-\mathrm{OCH}_{3}\right) ; 74.42$ (C-4'); 86.40 (C-2); 114.11, 129.02 (CH-Ph); 134.01 (C ipso Ph); 141.29 (C-4); 143.20 (C-5); $153.71(\mathrm{NC}=\mathrm{O}) ; 159.57$ (C ipso-Ph-OMe); 209.78 (C-3); IR (KBr): $\tilde{v}\left[\mathrm{~cm}^{-1}\right]=3452(\mathrm{O}-\mathrm{H}) ; 2965(\mathrm{C}-$ $\mathrm{H}) ; 1700(\mathrm{NC}=\mathrm{O}) ; 1669(\mathrm{C}=\mathrm{C}-\mathrm{C}=\mathrm{O}) ; \mathrm{ESI}-\mathrm{MS}: \mathrm{m} / \mathrm{z}=456.4\left[\left(\mathrm{M}+\mathrm{Na}^{+}\right)\right] ; \mathrm{C}_{25} \mathrm{H}_{39} \mathrm{NO}_{5}$ (433.28) Calcd. C 69.25, H 9.07, N 3.23; Found C 69.15, H 8.69, N 3.33 .

\section{(E)-3-[1-Hydroxy-1-(4-methoxyphenyl)methyl]-1,1,5,5-tetramethyl-2-oxo-3-hexenyl $N, N$ -} diisopropylcarbamate $((E)-11 d)$

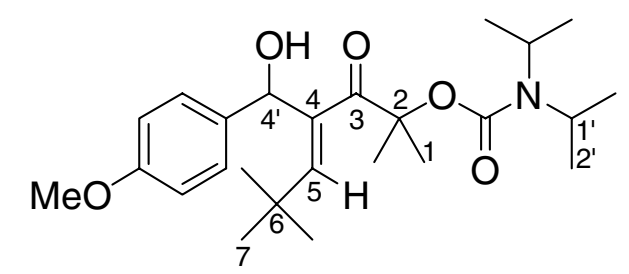

According to GEP 3, $154 \mathrm{mg}(0.51 \mathrm{mmol})$ of 8a resulted in $134 \mathrm{mg}(0.31 \mathrm{mmol}, 63 \%)(E)$-11d, colourless solid; $\mathrm{R}_{\mathrm{f}}=$ $0.65\left(\mathrm{Et}_{2} \mathrm{O} /\right.$ pentane $\left.=2 / 5\right) ; \mathrm{m} . \mathrm{p} .=97{ }^{\circ} \mathrm{C}($ from the melt $) ;{ }^{1} \mathrm{H}-\mathrm{NMR}(300 \mathrm{MHz}): \delta=1.15-1.35\left(\mathrm{br} \mathrm{m}, 12 \mathrm{H}, 1^{\prime}-\mathrm{H}_{3}\right) ; 1.22$

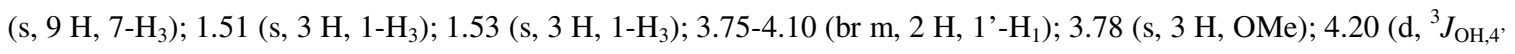
$=9.0 \mathrm{~Hz}, 1 \mathrm{H}, \mathrm{OH}) ; 5.85\left(\mathrm{~d},{ }^{3} J_{4^{\prime}, \mathrm{OH}}=9.0 \mathrm{~Hz}, 1 \mathrm{H}, 4^{\prime}-\mathrm{H}_{1}\right) ; 6.80-6.87(\mathrm{~m}, 2 \mathrm{H}, \mathrm{Ph}) ; 7.03\left(\mathrm{~s}, 1 \mathrm{H}, 5-\mathrm{H}_{1}\right) ; 7.32-7.40(\mathrm{~m}, 2$ $\mathrm{H}, \mathrm{Ph}$ ); ${ }^{13} \mathrm{C}-\mathrm{NMR}(75 \mathrm{MHz}): \delta=20.63,21.90,22.03$ (C-2'); 26.10, 26.26 (C-1); 31.21 (C-7); 33.44 (C-6); $45.79,46.77$ (C-1’); $55.19\left(\mathrm{Ar}^{\prime} \mathrm{OCH}_{3}\right) ; 69.92$ (C-4'); 83.65 (C-2); 113.31, 127.03 (CH-Ph); 135.54 (C ipso Ph); 137.17 (C-4); 151.60 (C-5); 153.95 (NC=O); 158.30 (C ipso-Ph-OMe); 202.38 (C-3); IR (KBr): $\widetilde{v}\left[\mathrm{~cm}^{-1}\right]=3467(\mathrm{O}-\mathrm{H}) ; 2999,2962$ $(\mathrm{C}-\mathrm{H}) ; 1704(\mathrm{NC}=\mathrm{O}) ; 1652(\mathrm{C}=\mathrm{C}-\mathrm{C}=\mathrm{O}) ; \mathrm{ESI}-\mathrm{MS}: \mathrm{m} / \mathrm{z}=890\left[\left(2 \mathrm{M}+\mathrm{Na}^{+}\right)\right] ; 456\left[\left(\mathrm{M}+\mathrm{Na}^{+}\right)\right] ; \mathrm{C}_{25} \mathrm{H}_{39} \mathrm{NO}_{5}(433.28) \mathrm{Calcd}$. C 69.25, H 9.07, N 3.23; Found C 69.00, H 9.00, N 3.15. 


\section{(Z)-3-[1-Hydroxy-1-(2-methylphenyl)methyl]-1,1,5,5-tetramethyl-2-oxo-3-hexenyl $N, N$ -}

diisopropylcarbamate $((Z)-10 \mathrm{e})$

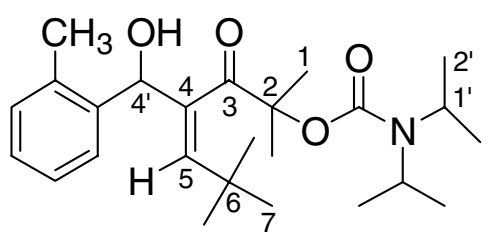

According to GEP 2, $108 \mathrm{mg}(0.36 \mathrm{mmol})$ of $\mathbf{8 a}$ resulted in $120 \mathrm{mg}(0.29 \mathrm{mmol}, 79 \%)(Z)-\mathbf{1 0 e}$, white solid; $\mathrm{R}_{\mathrm{f}}=0.65$ $\left(\mathrm{Et}_{2} \mathrm{O} /\right.$ pentane $\left.=2 / 5\right) ;$ m.p. $=132{ }^{\circ} \mathrm{C}($ from the melt $) ;{ }^{1} \mathrm{H}-\mathrm{NMR}(300 \mathrm{MHz}): \delta=0.98\left(\mathrm{~s}, 9 \mathrm{H}, 7-\mathrm{H}_{3}\right) ; 1.21-1.33\left(\mathrm{~d},{ }^{3} J_{2},{ }^{\prime},{ }^{\prime}=\right.$ $\left.6.8 \mathrm{~Hz}, 12 \mathrm{H}, 2^{\prime}-\mathrm{H}_{3}\right) ; 1.71$ (s, $\left.6 \mathrm{H}, 1-\mathrm{H}_{3}\right) ; 2.31$ (s, $\left.3 \mathrm{H}, \mathrm{Ar}-\mathrm{CH}_{3}\right) ; 3.31\left(\mathrm{~d},{ }^{3} J_{\mathrm{OH}, 4}=5.0 \mathrm{~Hz}, 1 \mathrm{H}, \mathrm{OH}\right) ; 3.70-4.15$ (br s, 2 $\left.\mathrm{H}, 1^{\prime}-\mathrm{H}_{1}\right) ; 5.13\left(\mathrm{~d},{ }^{4} J_{5,4^{\prime}}=1.2 \mathrm{~Hz}, 1 \mathrm{H}, 5-\mathrm{H}_{1}\right) ; 5.78\left(\mathrm{~d},{ }^{3} J_{4}, \mathrm{OH}=5.0 \mathrm{~Hz}, 1 \mathrm{H}, 4^{\prime}-\mathrm{H}_{1}\right) ; 7.07-7.24(\mathrm{~m}, 3 \mathrm{H}, \mathrm{Ph}) ; 7.42-7.51$ $(\mathrm{m}, 1 \mathrm{H}, \mathrm{Ph}) ;{ }^{13} \mathrm{C}-\mathrm{NMR}(75 \mathrm{MHz}): \delta=19.76\left(\mathrm{CH}_{3}-\mathrm{Ar}\right) ; 21.41$ (C-2'); 25.99, 27.14 (C-1); 30.57 (C-7); $33.84(\mathrm{C}-6)$; 46.63 (C-1'); 72.35 (C-4'); 85.83 (C-2); 126.49, 127.78, 128.01, 130.59 (CH-Ph); 136.19 (C ipso-Ph); 139.76, 139.81 (C-4, C ipso-Ph); 143.50 (C-5); 154.09 (NC=O); 209.19 (C-3); IR (KBr): $\tilde{v}$ [ $\left.\mathrm{cm}^{-1}\right]=3438(\mathrm{O}-\mathrm{H}) ; 2972(\mathrm{C}-\mathrm{H}) ; 1706$ $(\mathrm{NC}=\mathrm{O}) ; 1679(\mathrm{C}=\mathrm{C}-\mathrm{C}=\mathrm{O}) ; \mathrm{ESI}-\mathrm{MS}: \mathrm{m} / \mathrm{z}=440.3\left[\left(\mathrm{M}+\mathrm{Na}^{+}\right)\right] ; \mathrm{C}_{25} \mathrm{H}_{39} \mathrm{NO}_{4}$ (417.29) Calcd. C 71.91, H 9.41, N 3.35; Found C 71.87, H 9.25, N 3.26.

(E)-3-[1-Hydroxy-1-(2-methylphenyl)methyl]-1,1,5,5-tetramethyl-2-oxo-3-hexenyl $N, N$ diisopropylcarbamate $((E)-11 e)$

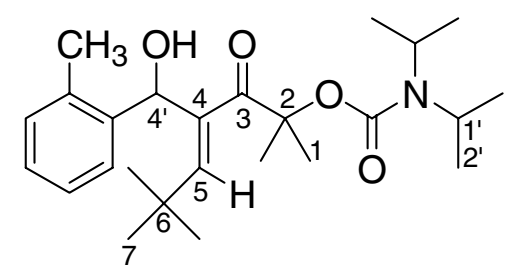

According to GEP 3, $148 \mathrm{mg}(0.49 \mathrm{mmol})$ of 8a resulted in $141 \mathrm{mg}(0.33 \mathrm{mmol}, 68 \%)(E)-\mathbf{1 1 e}$, colourless solid; $\mathrm{R}_{\mathrm{f}}=$ $0.65\left(\mathrm{Et}_{2} \mathrm{O} /\right.$ pentane $\left.=2 / 5\right) ; \mathrm{m} . \mathrm{p} .=88{ }^{\circ} \mathrm{C}($ from the melt $) ;{ }^{1} \mathrm{H}-\mathrm{NMR}(300 \mathrm{MHz}): \delta=1.07\left(\mathrm{~s}, 9 \mathrm{H}, 7-\mathrm{H}_{3}\right) ; 1.15-1.37(\mathrm{br} \mathrm{m}$, $12 \mathrm{H}, 2$ '- $\left.-\mathrm{H}_{3}\right) ; 1.57$ (s, $\left.3 \mathrm{H}, 1-\mathrm{H}_{3}\right) ; 1.63\left(\mathrm{~s}, 3 \mathrm{H}, 1-\mathrm{H}_{3}\right) ; 2.48$ (s, $\left.3 \mathrm{H}, \mathrm{Ar}-\mathrm{CH}_{3}\right) ; 3.68-4.18$ (br m, 2 H, 1'- $\left.\mathrm{H}_{1}\right) ; 4.18(\mathrm{~d}$, $\left.{ }^{3} J_{\mathrm{OH}, 4^{\prime}}=8.6 \mathrm{~Hz}, 1 \mathrm{H}, \mathrm{OH}\right) ; 5.92\left(\mathrm{~d},{ }^{3} J_{4}, \mathrm{OH}=8.6 \mathrm{~Hz}, 1 \mathrm{H}, 4^{\prime}-\mathrm{H}_{1}\right) ; 6.83\left(\mathrm{~d},{ }^{4} J_{5,7}=0.8 \mathrm{~Hz}, 1 \mathrm{H}, 5-\mathrm{H}_{1}\right) ; 7.06-7.13(\mathrm{~m}, 3 \mathrm{H}$, $\mathrm{Ph}) ; 7.30-7.38$ (m, $1 \mathrm{H}, \mathrm{Ph}) ;{ }^{13} \mathrm{C}-\mathrm{NMR}(75 \mathrm{MHz}): \delta=19.71\left(\mathrm{CH}_{3}-\mathrm{Ar}\right) ; 20.59,21.92,22.11(\mathrm{C}-2$ '); 26.15, $26.79(\mathrm{C}-1)$; 30.52 (C-7); 33.89 (C-6); 45.59, 46.83 (C-1'); 69.25 (C-4'); 83.89 (C-2); 125.40, 127.29, 128.01, 130.11 (CH-Ph); 136.73, 136.94, 140.84 (C-4, C ipso-Ph); $149.80(\mathrm{C}-5) ; 154.16(\mathrm{NC}=\mathrm{O}) ; 203.30(\mathrm{C}-3)$; IR $(\mathrm{KBr}): \tilde{v}\left[\mathrm{~cm}^{-1}\right]=3454(\mathrm{O}-$ $\mathrm{H}) ; 2966(\mathrm{C}-\mathrm{H}) ; 1686(\mathrm{NC}=\mathrm{O}) ; 1666(\mathrm{C}=\mathrm{C}-\mathrm{C}=\mathrm{O}) ; \mathrm{ESI}-\mathrm{MS}: \mathrm{m} / \mathrm{z}=440.4\left[\left(\mathrm{M}+\mathrm{Na}^{+}\right)\right] ; \mathrm{C}_{25} \mathrm{H}_{39} \mathrm{NO}_{4}$ (417.29) Calcd. C 71.91, H 9.41, N 3.35; Found C 72.02, H 9.45, N 3.31. 


\section{(Z)-3-(1-Hydroxy-1-naphth-2-ylmethyl)-1,1,5,5-tetramethyl-2-oxo-3-hexenyl $N, N$ -}

diisopropylcarbamate $((Z)-10 f)$

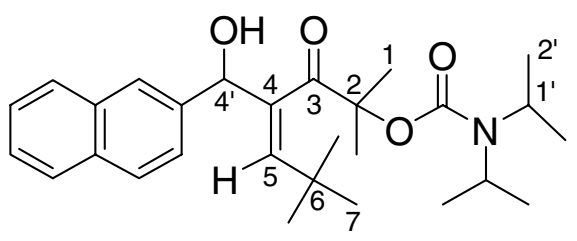

According to GEP 2, $139 \mathrm{mg}(0.46 \mathrm{mmol})$ of $\mathbf{8 a}$ resulted in $174 \mathrm{mg}(0.38 \mathrm{mmol}, 82 \%)(Z)-\mathbf{1 0 f}$, colourless liquid; $\mathrm{R}_{\mathrm{f}}=$ $0.43\left(\mathrm{Et}_{2} \mathrm{O} /\right.$ pentane $\left.=2 / 5\right) ;{ }^{1} \mathrm{H}-\mathrm{NMR}(300 \mathrm{MHz}): \delta=0.99\left(\mathrm{~s}, 9 \mathrm{H}, 7-\mathrm{H}_{3}\right) ; 1.26\left(\mathrm{t},{ }^{3} J_{2},{ }_{1},{ }^{\prime}=6.7 \mathrm{~Hz}, 12 \mathrm{H}, 2{ }^{\prime}-\mathrm{H}_{3}\right) ; 1.75$, $1.78\left(\mathrm{~s}, 6 \mathrm{H}, 1-\mathrm{H}_{3}\right) ; 3.22\left(\mathrm{~d},{ }^{3} J_{\mathrm{OH}, 4^{\prime}}=4.6 \mathrm{~Hz}, 1 \mathrm{H}, \mathrm{OH}\right) ; 3.82-4.15\left(\mathrm{br} \mathrm{m}, 2 \mathrm{H}, 1\right.$ '- $\left.\mathrm{H}_{1}\right) ; 5.35\left(\mathrm{~d},{ }^{4} J_{5,4^{4}}=1.0 \mathrm{~Hz}, 1 \mathrm{H}, 5-\mathrm{H}_{1}\right)$; $5.83\left(\mathrm{~d},{ }^{3} J_{4}, \mathrm{OH}=4.6 \mathrm{~Hz}, 1 \mathrm{H}, 4\right.$ '- $\left.\mathrm{H}_{1}\right) ; 7.41-7.50$ (m, $\left.3 \mathrm{H}, \mathrm{Naph}\right) ; 7.79-7.85$ (m, $\left.4 \mathrm{H}, \mathrm{Naph}\right) ;{ }^{13} \mathrm{C}-\mathrm{NMR}(75 \mathrm{MHz}): \delta=$ 21.07 (C-2'); 25.99, 26.81 (C-1); 30.17 (C-7); 33.57 (C-6); 46.26 (C-1'); 74.54, 74.61 (C-4'); 85.92 (C-2); 125.43, 125.94, 126.02, 127.66, 127.86, 128.16 (CH, Naph); 133.00, 133.29, 139.05, 140.82 (C ipso Naph, C-4); 144.18, 144.24 (C-5); $153.52(\mathrm{NC}=\mathrm{O}) ; 209.13(\mathrm{C}-3)$; IR (Film in $\left.\mathrm{Et}_{2} \mathrm{O}\right): \tilde{\boldsymbol{v}}$ [ $\left.\mathrm{cm}^{-1}\right]=3413(\mathrm{O}-\mathrm{H}) ; 2967,2869$ (C-H); 1699 $(\mathrm{NC}=\mathrm{O}) ; 1678(\mathrm{C}=\mathrm{C}-\mathrm{C}=\mathrm{O}) ; \mathrm{ESI}-\mathrm{MS}: \mathrm{m} / \mathrm{z}=454.4\left[\left(\mathrm{M}+\mathrm{H}^{+}\right)\right] ; 476.4\left[\left(\mathrm{M}+\mathrm{Na}^{+}\right)\right] ; \mathrm{C}_{28} \mathrm{H}_{39} \mathrm{NO}_{4}(453.29)$ Calcd. $\mathrm{C} 74.14, \mathrm{H}$ 8.67, N 3.09; Found C 73.81, H 8.37, N 3.02.

\section{(E)-3-(1-Hydroxy-1-naphth-2-ylmethyl)-1,1,5,5-tetramethyl-2-oxo-3-hexenyl $N, N$ -} diisopropylcarbamate $((E)-11 f)$

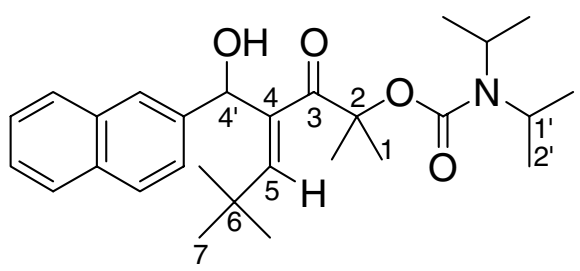

According to GEP 3, $150 \mathrm{mg}(0.50 \mathrm{mmol})$ of 8a resulted in $174 \mathrm{mg}(0.75 \mathrm{mmol}, 76 \%)(E)-\mathbf{1 1 f}$, colourless solid; $\mathrm{R}_{\mathrm{f}}=$ $0.43\left(\mathrm{Et}_{2} \mathrm{O} /\right.$ pentane $\left.=2 / 5\right) ;$ m.p. $=155{ }^{\circ} \mathrm{C}($ from the melt $) ;{ }^{1} \mathrm{H}-\mathrm{NMR}(300 \mathrm{MHz}): \delta=1.26\left(\mathrm{~s}, 9 \mathrm{H}, 7-\mathrm{H}_{3}\right) ; 1.20-1.35(\mathrm{br}$ m, 12 H, 2'- $\left.-\mathrm{H}_{3}\right) ; 1.51$ (s, $\left.3 \mathrm{H}, 1-\mathrm{H}_{3}\right) ; 1.53$ (s, $\left.3 \mathrm{H}, 1-\mathrm{H}_{3}\right) ; 3.72-4.11$ (br m, $2 \mathrm{H}, 1$ '- $\left.-\mathrm{H}_{1}\right) ; 6.05$ (s, 1 H, 4'- $\mathrm{H}_{1}$ ); 7.12 (s, 1 H, 5-H ) $_{1}$ 7.30-7.45 (m, 2 H, Naph); 7.6 (m, 1 H, Naph); 7.75-7.85 (m, 3 H, Naph); 7.95 (s, 1 H, Naph); ${ }^{13} \mathrm{C}-\mathrm{NMR}(75$ MHz): $\delta=20.69,21.89,21.99$ (C-2'); 26.14, 26.19 (C-1); 31.26 (C-7); 33.51 (C-6); 45.87, 46.80 (C-1'); 70.34 (C-4'); 83.63 (C-2); 124.32, 124.54, 125.24, 125.57, 127.33, 127.51, 128.19 (CH-Naph); 132.51, 140.84 (C ipso Naph); 137.06 (C-4); $152.16(\mathrm{C}-5) ; 153.95(\mathrm{NC}=\mathrm{O}) ; 202.26(\mathrm{C}-3) ; \mathrm{IR}(\mathrm{KBr}): \tilde{v} \quad\left[\mathrm{~cm}^{-1}\right]=3498(\mathrm{O}-\mathrm{H}) ; 2994,2935,2872(\mathrm{C}-\mathrm{H}) ; 1697$ $(\mathrm{NC}=\mathrm{O}) ; 1656,1626(\mathrm{C}=\mathrm{C}-\mathrm{C}=\mathrm{O}) ; \mathrm{ESI}-\mathrm{MS}: \mathrm{m} / \mathrm{z}=476.7\left[\left(\mathrm{M}+\mathrm{Na}^{+}\right)\right] ; \mathrm{C}_{28} \mathrm{H}_{39} \mathrm{NO}_{4}$ (453.29), Calcd. C 74.14, $\mathrm{H} \mathrm{8.67,} \mathrm{N}$ 3.09; Found C 74.01, H 8.50, N 2.99. 


\section{(Z)-3-[1-(4-Chlorophenyl)-1-hydroxymethyl]-1,1,5,5-tetramethyl-2-oxo-3-hexenyl $N, N$ -}

diisopropylcarbamate $((Z)-10 \mathrm{~g})$<smiles>CC(C)N(C(=O)OC(C)(C)C(=O)C(=CC(C)(C)C)C(O)c1ccc(Cl)cc1)C(C)C</smiles>

According to GEP 2, $122 \mathrm{mg}(0.41 \mathrm{mmol})$ of $\mathbf{8 a}$ resulted in $148 \mathrm{mg}(0.34 \mathrm{mmol}, 82 \%)(Z)-\mathbf{1 0 g}$, white solid; $\mathrm{R}_{\mathrm{f}}=0.50$ $\left(\mathrm{Et}_{2} \mathrm{O} /\right.$ pentane $\left.=2 / 5\right) ; \mathrm{m} . \mathrm{p} .=128{ }^{\circ} \mathrm{C}$ (from the melt); ${ }^{1} \mathrm{H}-\mathrm{NMR}(300 \mathrm{MHz}): \delta=1.00\left(\mathrm{~s}, 9 \mathrm{H}, 7-\mathrm{H}_{3}\right) ; 1.24\left(\mathrm{dd},{ }^{4} J_{2}, 1,{ }^{\prime}=\right.$ $\left.6.8 \mathrm{~Hz}, 12 \mathrm{H}, 2^{\prime}-\mathrm{H}_{3}\right) ; 1.70,1.73$ (s, $\left.6 \mathrm{H}, 1-\mathrm{H}_{3}\right) ; 3.45\left(\mathrm{~d},{ }^{3} J_{\mathrm{OH}, 4^{\prime}}=4.0 \mathrm{~Hz}, 1 \mathrm{H}, \mathrm{OH}\right) ; 3.90$ (br s, $\left.2 \mathrm{H}, 1{ }^{\prime}-\mathrm{H}_{1}\right) ; 5.31\left(\mathrm{~d},{ }^{4} J_{5,4^{\prime}}\right.$ $\left.=1.0 \mathrm{~Hz}, 1 \mathrm{H}, 5-\mathrm{H}_{1}\right) ; 5.60\left(\mathrm{~d},{ }^{3} J_{4}, \mathrm{OH}=4.0 \mathrm{~Hz}, 1 \mathrm{H}, 4^{\prime}-\mathrm{H}_{1}\right) ; 7.30(\mathrm{~m}, 4 \mathrm{H}, \mathrm{Ph}) ;{ }^{13} \mathrm{C}-\mathrm{NMR}(75 \mathrm{MHz}): \delta=21.39\left(\mathrm{C}-2^{\prime}\right)$; 26.53, 27.13 (C-1); 30.47 (C-7); 33.91 (C-6); 46.66 (C-1'); 74.45 (C-4'); 86.14 (C-2); 128.72, 128.91 (CH-Ph); 133.64, 140.64, 140.96 (C ipso-Ph, C-4); 144.20 (C-5); $153.95(\mathrm{NC}=\mathrm{O}) ; 209.30(\mathrm{C}-3) ; \mathrm{IR}(\mathrm{KBr}): \widetilde{v}\left[\mathrm{~cm}^{-1}\right]=3426(\mathrm{O}-\mathrm{H})$; 2960 (C-H); $1704(\mathrm{NC}=\mathrm{O}) ; 1673(\mathrm{C}=\mathrm{C}-\mathrm{C}=\mathrm{O})$; ESI-MS: $\mathrm{m} / \mathrm{z}=460.4,462.4\left[\left(\mathrm{M}+\mathrm{Na}^{+}\right)\right] ; \mathrm{C}_{24} \mathrm{H}_{36} \mathrm{ClNO}_{4}(437.23)$ Calcd. C 65.81, H 8.28, N 3.20; Found C 65.93, H 8.08, N 3.19.

\section{(E)-3-[1-(4-Chlorophenyl)-1-hydroxymethyl]-1,1,5,5-tetramethyl-2-oxo-3-hexenyl $N, N$ -} diisopropylcarbamate $((E)-11 g)$

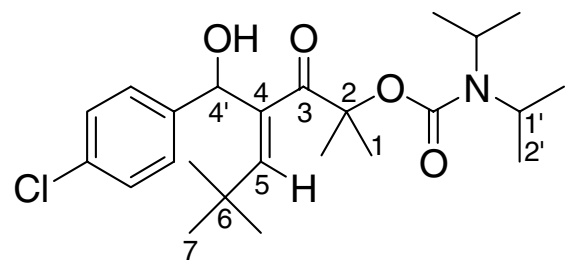

According to GEP 3, $149 \mathrm{mg}(0.50 \mathrm{mmol})$ of 8a resulted in $110 \mathrm{mg}(0.25 \mathrm{mmol}, 50 \%)(E)-\mathbf{1 1 g}$, colourless liquid which became a solid in the refrigerator. Melting point was not recorded as it melts when it is kept outside; $\mathrm{R}_{\mathrm{f}}=0.53$ $\left(\mathrm{Et}_{2} \mathrm{O} /\right.$ pentane $\left.=2 / 5\right) ;{ }^{1} \mathrm{H}-\mathrm{NMR}(300 \mathrm{MHz}): \delta=1.23\left(\mathrm{~s}, 9 \mathrm{H}, 7-\mathrm{H}_{3}\right) ; 1.19-1.32\left(\mathrm{br} \mathrm{m}, 12 \mathrm{H}, 2\right.$ '- $\left.\mathrm{H}_{3}\right) ; 1.53\left(\mathrm{~s}, 6 \mathrm{H}, 1-\mathrm{H}_{3}\right)$; 3.70-3.88 (br m, $1 \mathrm{H}, 1$ ''- $\left.\mathrm{H}_{1}\right)$; 3.90-4.07 (m, $1 \mathrm{H}, 1$ ' $\left.-\mathrm{H}_{1}\right) ; 4.23\left(\mathrm{~d},{ }^{3} J_{\mathrm{OH}, 4^{\prime}}=8.7 \mathrm{~Hz}, 1 \mathrm{H}, \mathrm{OH}\right) ; 5.84\left(\mathrm{~d},{ }^{3} J_{4}, \mathrm{OH}=8.7 \mathrm{~Hz}, 1\right.$ $\left.\mathrm{H}, 4^{\prime}-\mathrm{H}_{1}\right) ; 7.08$ (s, $\left.1 \mathrm{H}, 5-\mathrm{H}_{1}\right) ; 7.25-7.31$ (m, $\left.2 \mathrm{H}, \mathrm{Ph}\right) ; 7.37-7.41$ (m, $\left.2 \mathrm{H}, \mathrm{Ph}\right) ;{ }^{13} \mathrm{C}-\mathrm{NMR}(75 \mathrm{MHz}): \delta=20.59,20.65$, 21.86, 21.98 (C-2’); 26.17 (C-1); 31.21 (C-7); 33.39 (C-6); 45.86, 46.78 (C-1'); 69.61 (C-4'); 83.57 (C-2); 127.15, 127.95, (CH-Ph); 132.17, 141.94 (C ipso-Ph); 136.70 (C-4); 152.32 (C-5); 153.97 (NC=O); 202.16 (C-3); IR (Film): $\tilde{v}\left[\mathrm{~cm}^{-1}\right]=3456(\mathrm{O}-\mathrm{H}) ; 2968,2872(\mathrm{C}-\mathrm{H}) ; 1693(\mathrm{NC}=\mathrm{O}) ; 1661(\mathrm{C}=\mathrm{C}-\mathrm{C}=\mathrm{O}) ; \mathrm{ESI}-\mathrm{MS}: \mathrm{m} / \mathrm{z}=460.3,462.3\left[\left(\mathrm{M}^{+} \mathrm{Na}^{+}\right)\right]$; $\mathrm{C}_{24} \mathrm{H}_{36} \mathrm{ClNO}_{4}(437.23)$ Calcd. C 65.81, H 8.28, N 3.20; Found C 65.58, H 8.37, N 3.05. 


\section{(Z)-3-(1-Furyl-1-hydroxymethyl)-1,1,5,5-tetramethyl-2-oxo-3-hexenyl $N, N$ -}

diisopropylcarbamate $((Z)-10 h)$

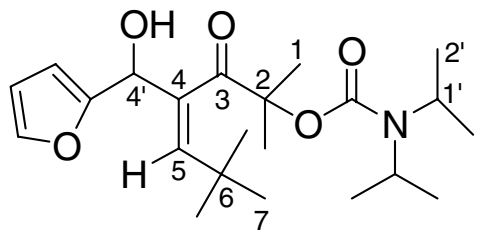

According to GEP 2, $150 \mathrm{mg}(0.50 \mathrm{mmol})$ of $\mathbf{8 a}$ resulted in $124 \mathrm{mg}(0.31 \mathrm{mmol}, 63 \%)(Z)$-10h, light yellow solid; $\mathrm{R}_{\mathrm{f}}$ $=0.50\left(\mathrm{Et}_{2} \mathrm{O} /\right.$ pentane $\left.=2 / 5\right) ; \mathrm{m} . \mathrm{p} .=114{ }^{\circ} \mathrm{C}$ (from the melt); ${ }^{1} \mathrm{H}-\mathrm{NMR}(300 \mathrm{MHz}): \delta=1.06\left(\mathrm{~s}, 9 \mathrm{H}, 7-\mathrm{H}_{3}\right) ; 1.23(\mathrm{~d}$, $\left.{ }^{3} J_{2}{ }^{\prime}, 1^{\prime}=6.8 \mathrm{~Hz}, 12 \mathrm{H}, 2{ }^{\prime}-\mathrm{H}_{3}\right) ; 1.65,1.70\left(\mathrm{~s}, 6 \mathrm{H}, 1-\mathrm{H}_{3}\right) ; 2.80\left(\mathrm{~d},{ }^{3} J_{\mathrm{OH}, 4}=6.0 \mathrm{~Hz}, 1 \mathrm{H}, \mathrm{OH}\right) ; 3.60-4.22\left(\mathrm{br} \mathrm{m}, 2 \mathrm{H}, 1\right.$ ' - $\left.\mathrm{H}_{1}\right)$; $5.54\left(\mathrm{~d},{ }^{4} J_{5,4}=1.1 \mathrm{~Hz}, 1 \mathrm{H}, 5-\mathrm{H}_{1}\right) ; 5.69\left(\mathrm{~d},{ }^{3} J_{4, \mathrm{OH}}=6.0 \mathrm{~Hz}, 1 \mathrm{H}, 1-\mathrm{H}_{4}\right)$; 6.29-6.34 (m, $\left.2 \mathrm{H}, \mathrm{Ar}\right) ; 7.36-7.38(\mathrm{~m}, 1 \mathrm{H}, \mathrm{Ar})$; ${ }^{13}$ C-NMR (75 MHz): $\delta=21.01$ (C-2'); 25.91, 26.30 (C-1); 30.25 (C-7); 33.53 (C-6); 46.18 (C-1'); 68.52 (C-4'); 85.79 (C-2); 108.47, 110.37, 142.32, 154.39 (Ar); 138.36 (C-4); 143.95 (C-5); 153.21 (NC=O); 208.32 (C-3); IR (KBr): $\tilde{\boldsymbol{V}}$ $\left.\left[\mathrm{cm}^{-1}\right]=3403(\mathrm{O}-\mathrm{H}) ; 2967,2876(\mathrm{C}-\mathrm{H}) ; 1699(\mathrm{NC}=\mathrm{O}) ; 1639(\mathrm{C}=\mathrm{C}-\mathrm{C}=\mathrm{O}) ; \mathrm{ESI}-\mathrm{MS}: \mathrm{m} / \mathrm{z}=416.6\left[\left(\mathrm{M}^{+\mathrm{Na}}\right)^{+}\right)\right]$ $\mathrm{C}_{22} \mathrm{H}_{35} \mathrm{NO}_{5}$ (393.25); Calcd. C 67.15, H 8.96, N 3.56, Found C 66.85, H 8.95, N 3.50.

\section{(Z)-3-[1-Hydroxy-1-(2,4,6-trimethoxyphenyl)methyl]-1,1,5,5-tetramethyl-2-oxo-3-hexenyl}

\section{$N, N$-diisopropylcarbamate $((Z)-10 i)$}

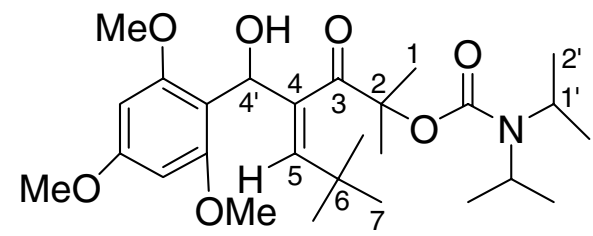

According to GEP 2, $119 \mathrm{mg}(0.40 \mathrm{mmol})$ of 8a resulted in $132 \mathrm{mg}(0.27 \mathrm{mmol}, 67 \%)(Z)-10 i$, white solid; $\mathrm{R}_{\mathrm{f}}=0.45$ $($ EtOAc/pentane $=2 / 5) ;$ m.p. $=128{ }^{\circ} \mathrm{C}($ from the melt $) ;{ }^{1} \mathrm{H}-\mathrm{NMR}(300 \mathrm{MHz}): \delta=1.03\left(\mathrm{~s}, 9 \mathrm{H}, 7-\mathrm{H}_{3}\right) ; 1.25\left(\mathrm{~d},{ }^{3} J_{2},{ }_{1},=\right.$ $6.8 \mathrm{~Hz}, 12 \mathrm{H}, 2$ '- $\left.\mathrm{H}_{3}\right) ; 1.67$ (s, $\left.3 \mathrm{H}, 1-\mathrm{H}_{3}\right) ; 1.71\left(\mathrm{~s}, 3 \mathrm{H}, 1-\mathrm{H}_{3}\right) ; 3.26\left(\mathrm{~d},{ }^{3} J_{\mathrm{OH}, 4}=5.5 \mathrm{~Hz}, 1 \mathrm{H}, \mathrm{OH}\right) ; 3.58-4.10(\mathrm{br} \mathrm{m}, 2 \mathrm{H}$, $\left.1^{\prime}-\mathrm{H}_{1}\right) ; 3.79,3.83,3.89$ (s, $\left.9 \mathrm{H}, \mathrm{Ar}-\mathrm{OCH}_{3}\right) ; 5.31\left(\mathrm{~d},{ }^{4} J_{5,4^{\prime}}=1.4 \mathrm{~Hz}, 1 \mathrm{H}, 5-\mathrm{H}_{1}\right) ; 5.90\left(\mathrm{dd},{ }^{3} J_{4}, \mathrm{OH}=5.5 \mathrm{~Hz},{ }^{4} J_{4}, 5=1.4 \mathrm{~Hz}\right.$, $\left.1 \mathrm{H}, 4^{\prime}-\mathrm{H}_{1}\right) ; 6.50$ (s,1 H, CH-Ph); 6.96 (s, $\left.1 \mathrm{H}, \mathrm{CH}-\mathrm{Ph}\right) ;{ }^{13} \mathrm{C}-\mathrm{NMR}(75 \mathrm{MHz}): \delta=21.38$ (C-2'); 25.92, 26.69 (C-1); 30.80 (C-7); 33.72 (C-6); 46.49 (C-1’); 56.54, $56.73\left(\mathrm{Ar}-\mathrm{OCH}_{3}\right)$; 69.41 (C-4'); 85.81 (C-2); 97.77, 112.89 (CH-Ph); 140.30 (C-4); 142.17 (C-5); 143.50, 149.49, 151.46 (C ipso-Ph); $153.96(\mathrm{NC}=\mathrm{O}) ; 209.22(\mathrm{C}-3) ; \mathrm{IR}(\mathrm{KBr}): \tilde{\boldsymbol{V}}\left[\mathrm{cm}^{-1}\right]=$ $3473(\mathrm{O}-\mathrm{H}) ; 2964(\mathrm{C}-\mathrm{H}) ; 1705(\mathrm{NC}=\mathrm{O}) ; 1678(\mathrm{C}=\mathrm{C}-\mathrm{C}=\mathrm{O}) ; \mathrm{ESI}-\mathrm{MS}: \mathrm{m} / \mathrm{z}=516.5$ [(M+Na+$)] ; \mathrm{C}_{27} \mathrm{H}_{43} \mathrm{NO}_{7}(493.30)$ Calcd. C 65.69, H 8.78, N 2.84; Found C 65.40, H 8.66, N 2.73. 


\section{(E)-3-[1-Hydroxy-1-(2,4,6-trimethoxyphenyl)methyl]-1,1,5,5-tetramethyl-2-oxo-3-hexenyl} $N, N$-diisopropylcarbamate $((E)-11 i)$

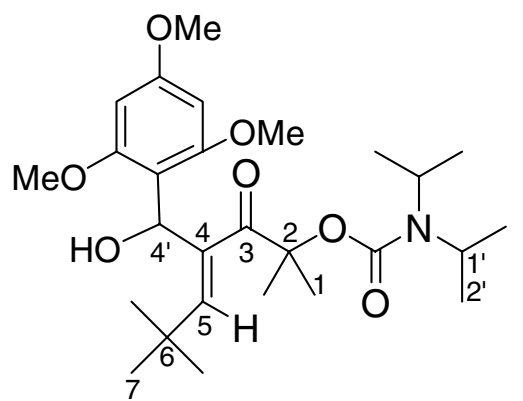

According to GEP 3, $151 \mathrm{mg}(0.50 \mathrm{mmol})$ of 8a resulted in $157 \mathrm{mg}(0.32 \mathrm{mmol}, 63 \%)(E)$-11i, colourless solid; $\mathbf{R}_{\mathrm{f}}=$ $0.45\left(\mathrm{Et}_{2} \mathrm{O} /\right.$ pentane $\left.=3 / 5\right) ;$ m.p. $=127{ }^{\circ} \mathrm{C}$ (from the melt); ${ }^{1} \mathrm{H}-\mathrm{NMR}(300 \mathrm{MHz}): \delta=1.15\left(\mathrm{~s}, 9 \mathrm{H}, 7-\mathrm{H}_{3}\right) ; 1.10-1.37(\mathrm{br}$ m, 12 H, 2'- $\left.\mathrm{H}_{3}\right) ; 1.46$ (s, $\left.3 \mathrm{H}, 1-\mathrm{H}_{3}\right) ; 1.50$ (s, $\left.3 \mathrm{H}, 1-\mathrm{H}_{3}\right) ; 3.58-4.10$ (br m, $\left.2 \mathrm{H}, 1^{\prime}-\mathrm{H}_{1}\right) ; 3.82,3.86,3.88$ (s, 9 H, OCH ${ }_{3}^{-}$ $\mathrm{Ph}) ; 4.42\left(\mathrm{~d},{ }^{3} J_{\mathrm{OH}, 4^{4}}=8.9 \mathrm{~Hz}, 1 \mathrm{H}, \mathrm{OH}\right) ; 6.13\left(\mathrm{~d},{ }^{3} J_{4^{\prime}, \mathrm{OH}}=8.9 \mathrm{~Hz}, 1 \mathrm{H}, 4{ }^{\prime}-\mathrm{H}_{1}\right) ; 6.48(\mathrm{~s}, 1 \mathrm{H}, \mathrm{CH}-\mathrm{Ph}) ; 6.70\left(\mathrm{~d},{ }^{4} J_{5,7}=0.8\right.$ $\mathrm{Hz}, 1 \mathrm{H}, 5-\mathrm{H1}$ ); 7.16 (s, $1 \mathrm{H}, \mathrm{CH}-\mathrm{Ph}) ;{ }^{13} \mathrm{C}-\mathrm{NMR}$ (75 MHz): $\delta=20.55,21.91$ (C-2'); 26.07 (C-1); 30.63 (C-7); 33.93 (C-6); 45.62, 46.71 (C-1'); 56.14, 56.38, 56.65 ( $\left.\mathrm{Ar}^{-} \mathrm{OCH}_{3}\right) ; 66.05$ (C-4'); 83.63 (C-2); 97.25, 113.41 (CH-Ph); 123.53, 142.87, 148.68, 150.99 (C ipso-Ph); $137.16(\mathrm{C}-4) ; 154.02(\mathrm{NC}=\mathrm{O}) ; 203.97(\mathrm{C}-3) ; \mathrm{IR}(\mathrm{KBr}): \tilde{V}\left[\mathrm{~cm}^{-1}\right]=3510(\mathrm{O}-\mathrm{H})$; 2996, $2960(\mathrm{C}-\mathrm{H}) ; 1692(\mathrm{NC}=\mathrm{O}) ; 1661(\mathrm{C}=\mathrm{C}-\mathrm{C}=\mathrm{O})$; ESI-MS: m/z = $516\left[\left(\mathrm{M}+\mathrm{Na}^{+}\right)\right] ; \mathrm{C}_{27} \mathrm{H}_{43} \mathrm{NO}_{7}$ (493.30) Calcd. C 65.69, H 8.78, N 2.84; Found C 65.54, H 8.74, N 2.62.

\section{(5E)-3-[(Z)-2,2-Dimethylpropylidene]-4-hydroxy-1,1,5-trimethyl-2-oxo-6-phenylhex-5-enyl}

\section{$N, N$-diisopropylcarbamate $((Z)-10 j)$}

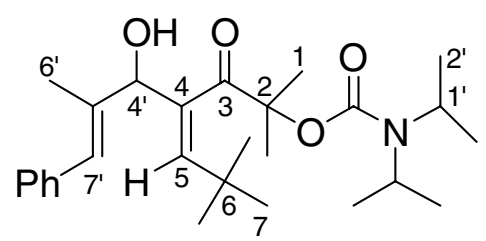

According to GEP 2, $137 \mathrm{mg}(0.46 \mathrm{mmol})$ of $\mathbf{8 a}$ resulted in $159 \mathrm{mg}(0.35 \mathrm{mmol}, 78 \%)(Z)-\mathbf{1 0 j}$, colourless liquid which became a solid on long standing; $\mathrm{R}_{\mathrm{f}}=0.65\left(\mathrm{Et}_{2} \mathrm{O} /\right.$ pentane $\left.=2 / 5\right) ; \mathrm{m} . \mathrm{p} .=110{ }^{\circ} \mathrm{C}$ (from the melt); ${ }^{1} \mathrm{H}-\mathrm{NMR}(300 \mathrm{MHz})$ : $\delta=1.07\left(\mathrm{~s}, 9 \mathrm{H}, 7-\mathrm{H}_{3}\right) ; 1.24\left(\mathrm{~d},{ }^{3} J_{2^{\prime}, 1^{\prime}}=6.9 \mathrm{~Hz}, 12 \mathrm{H}, 2^{\prime}-\mathrm{H}_{3}\right) ; 1.74\left(\mathrm{~s}, 3 \mathrm{H}, 1-\mathrm{H}_{3}\right) ; 1.76\left(\mathrm{~s}, 3 \mathrm{H}, 1-\mathrm{H}_{3}\right) ; 1.82\left(\mathrm{~d},{ }^{4} J_{6^{\prime}, 7}{ }^{\prime}=1.2\right.$ $\left.\mathrm{Hz}, 3 \mathrm{H}, 6{ }^{\prime}-\mathrm{H}_{3}\right) ; 2.82\left(\mathrm{~d},{ }^{3} J_{\mathrm{OH}, 4}=4.0 \mathrm{~Hz}, 1 \mathrm{H}, \mathrm{OH}\right) ; 3.70-4.10$ (br m, $\left.2 \mathrm{H}, 1^{\prime}-\mathrm{H}_{1}\right)$; $5.02\left(\mathrm{~d},{ }^{3} J_{4}\right.$, OH $\left.=4.0 \mathrm{~Hz}, 1 \mathrm{H}, 4^{\prime}-\mathrm{H}_{1}\right)$; $5.64\left(\mathrm{~d},{ }^{4} J_{5,4^{\prime}}=1.0 \mathrm{~Hz}, 1 \mathrm{H}, 5-\mathrm{H}_{1}\right) ; 6.64\left(\mathrm{~s}, 1 \mathrm{H}, 7^{\prime}-\mathrm{H}_{1}\right) ; 7.19-7.36(\mathrm{~m}, 5 \mathrm{H}, \mathrm{Ph}) ;{ }^{13} \mathrm{C}-\mathrm{NMR}(75 \mathrm{MHz}): \delta=15.16(\mathrm{C}-6$ '); 21.40 (C-2'); 26.58, 26.89 (C-1); 30.62 (C-7); 33.92 (C-6); 46.54 (C-1'); 77.73 (C-4'); 86.44 (C-2); 126.89, 127.84, 128.49 (CH-Ph); 129.38 (C-6’); 137.94, 138.01, 138.95 (C-4, C-5', C ipso-Ph); 143.13 (C-5); 153.65 (NC=O); 209.72 (C-3); IR (KBr): $\tilde{v}\left[\mathrm{~cm}^{-1}\right]=3466(\mathrm{O}-\mathrm{H}) ; 3008,2967(\mathrm{C}-\mathrm{H}) ; 1695(\mathrm{NC}=\mathrm{O}) ; 1676(\mathrm{C}=\mathrm{C}-\mathrm{C}=\mathrm{O}) ; \mathrm{ESI}-\mathrm{MS}: \mathrm{m} / \mathrm{z}=466.4$ $\left[\left(\mathrm{M}+\mathrm{Na}^{+}\right)\right] ; \mathrm{C}_{27} \mathrm{H}_{41} \mathrm{NO}_{4}(443.30)$ Calcd. C 73.10, H 9.32, N 3.16, Found C 73.13, H 9.26, N 3.03. 
(5E)-3-[(E)-2,2-Dimethylpropylidene]-4-hydroxy-1,1,5-trimethyl-2-oxo-6-phenylhex-5-enyl $N, N$-diisopropylcarbamate $((E)-\mathbf{1 1} \mathbf{j})$

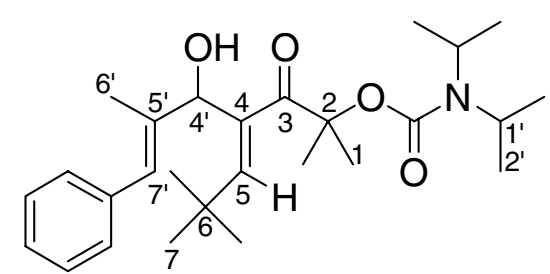

According to GEP 3, $150 \mathrm{mg}(0.50 \mathrm{mmol})$ of 8a resulted in $160 \mathrm{mg}(0.36 \mathrm{mmol}, 71 \%)(E)-\mathbf{1 1} \mathbf{j}$, colourless liquid; $\mathrm{R}_{\mathrm{f}}=$ $0.65\left(\mathrm{Et}_{2} \mathrm{O} /\right.$ pentane $\left.=2 / 5\right) ;{ }^{1} \mathrm{H}-\mathrm{NMR}(300 \mathrm{MHz}): \delta=1.15-1.40\left(\right.$ br m, $\left.12 \mathrm{H}, 2{ }^{\prime}-\mathrm{H}_{3}\right) ; 1.25\left(\mathrm{~s}, 9 \mathrm{H}, 7-\mathrm{H}_{3}\right) ; 1.58(\mathrm{~s}, 3 \mathrm{H}, 1-$ $\left.\mathrm{H}_{3}\right) ; 1.61$ (s, $\left.3 \mathrm{H}, 1-\mathrm{H}_{3}\right) ; 1.92\left(\mathrm{t},{ }^{4} J_{6^{\prime}, 7}\right.$ = $\left.1.1 \mathrm{~Hz}, 3 \mathrm{H}, 6{ }^{\prime}-\mathrm{H}_{3}\right) ; 3.73-4.09$ (br, m, $\left.2 \mathrm{H}, 1^{\prime}-\mathrm{H}_{1}\right)$; $4.22\left(\mathrm{~d},{ }^{3} J_{\mathrm{OH}, 4}=8.7 \mathrm{~Hz}, 1 \mathrm{H}\right.$, $\mathrm{OH}) ; 5.28\left(\mathrm{~d},{ }^{3} J_{4^{\prime}, \mathrm{OH}}=8.7 \mathrm{~Hz}, 1 \mathrm{H}, 4^{\prime}-\mathrm{H}_{1}\right) ; 6.62\left(\mathrm{t},{ }^{4} J_{7^{\prime}, 6^{\prime}}=1.1 \mathrm{~Hz}, 1 \mathrm{H}, 7^{\prime}-\mathrm{H}_{1}\right) ; 6.87\left(\mathrm{~s}, 1 \mathrm{H}, 5-\mathrm{H}_{1}\right) ; 7.13-7.37(\mathrm{~m}, 5 \mathrm{H}$, $\mathrm{Ph}) ;{ }^{13} \mathrm{C}-\mathrm{NMR}$ (75 MHz): $\delta=16.17$ (C-6’); 20.98, 22.33 (C-2'); 26.86, 27.04 (C-1); 31.54 (C-7); 33.96 (C-6); 46.11 , 47.13 (C-1'); 73.30 (C-4'); 84.01 (C-2); 124.64, 126.16, 128.20 (CH-Ph); 129.51 (C-7'); 137.35 (C-4); 139.11, 139.42 (C-5', C ipso-Ph); 151.57 (C-5); 154.39 (NC=O); 203.29 (C-3); IR (Film): $\tilde{V} \quad\left[\mathrm{~cm}^{-1}\right]=3444$ (O-H); 2967, 2872 (C-H); $1693(\mathrm{NC}=\mathrm{O}) ; 1650(\mathrm{C}=\mathrm{C}-\mathrm{C}=\mathrm{O})$; ESI-MS: m/z = 466.8 [(M+Na+ $)] ; \mathrm{C}_{27} \mathrm{H}_{41} \mathrm{NO}_{4}(443.30)$ Calcd. C 73.10, H 9.32, N 3.16; Found C 72.82, H 9.37, N 3.09.

\section{(Z)-3-(1-Hydroxy-1-naphth-2-ylmethyl)-1,1-dimethyl-2-oxo-3-pentenyl $N, N$ -}

diisopropylcarbamate $((Z)-15 a)$

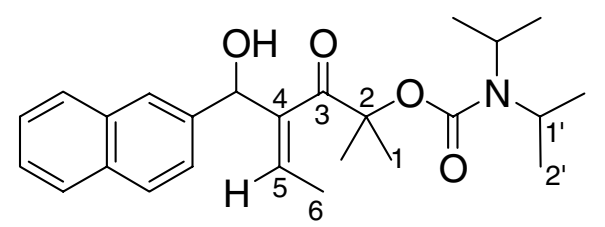

According to GEP 3, $198 \mathrm{mg}(0.77 \mathrm{mmol})$ of $8 \mathbf{b}$ resulted in $199 \mathrm{mg}(0.48 \mathrm{mmol}, 63 \%)(Z)-\mathbf{1 5 a}$, colourless solid; $\mathrm{R}_{\mathrm{f}}=$ $0.6\left(\mathrm{Et}_{2} \mathrm{O} /\right.$ pentane $\left.=1 / 2\right)$; m.p. $=124{ }^{\circ} \mathrm{C}$ (from the melt); ${ }^{1} \mathrm{H}-\mathrm{NMR}(300 \mathrm{MHz}): \delta=1.25\left(\mathrm{br} \mathrm{s}, 12 \mathrm{H}, 2^{\prime}-\mathrm{H}_{3}\right) ; 1.62,1.63$ $\left(\mathrm{dd},{ }^{3} J_{6,5}=7.2 \mathrm{~Hz}, 3 \mathrm{H}, 6-\mathrm{H}_{3}\right) ; 1.66\left(\mathrm{~s}, 3 \mathrm{H}, 1-\mathrm{H}_{3}\right) ; 1.76$ (s, $\left.3 \mathrm{H}, 1-\mathrm{H}_{3}\right) ; 3.70-4.05$ (br s, $2 \mathrm{H}, 1$ ' $\left.-\mathrm{H}_{1}\right) ; 4.30\left(\mathrm{~d},{ }^{3} J_{\mathrm{OH}, 4}{ }^{\prime}=4.4\right.$ $\mathrm{Hz}, 1 \mathrm{H}, \mathrm{OH}) ; 5.20-5.28\left(\mathrm{dq},{ }^{3} J_{5,6}=7.2 \mathrm{~Hz}, 1 \mathrm{H}, 5-\mathrm{H}_{1}\right) ; 5.82\left(\mathrm{~d},{ }^{3} J_{4}\right.$, OH $=4.0 \mathrm{~Hz}, 1 \mathrm{H}, 4$ '- $\left.\mathrm{H}_{1}\right) ; 7.43-7.48$ (m, $\left.3 \mathrm{H}, \mathrm{Naph}\right)$; 7.75-7.91 (m, 4 H, Naph); ${ }^{13}$ C-NMR (75 MHz): $\delta=15.69$ (C-6); 20.67, 21.48 (C-2'); 25.38, 25.92 (C-1); 46.63 (C-1'); 74.27 (C-4'); 83.72 (C-2); 125.56, 125.75, 125.96, 127.62, 128.12 (CH-Naph); 129.39 (C-5); 132.91, 133.31, 139.12 (C ipso Naph); 146.18 (C-4); 154.65 (NC=O); 208.02 (C-3); IR (KBr): $\tilde{v} \quad\left[\mathrm{~cm}^{-1}\right]=3430(\mathrm{O}-\mathrm{H}) ; 2982,29672933$ (C-H); $1679(\mathrm{NC}=\mathrm{O}) ; 1632,1603(\mathrm{C}=\mathrm{C}-\mathrm{C}=\mathrm{O}) ; \mathrm{ESI}-\mathrm{MS}: \mathrm{m} / \mathrm{z}=412.7\left[\left(\mathrm{M}+\mathrm{H}^{+}\right)\right] ; 434.6\left[\left(\mathrm{M}+\mathrm{Na}^{+}\right)\right] ; \mathrm{C}_{25} \mathrm{H}_{33} \mathrm{NO}_{4}(411.24) \mathrm{Calcd}$. C 72.96, H 8.08, N 3.40; Found C 72.96, H 8.33, N 2.94. 


\section{(Z)-3-[1-(4-Bromophenyl)-1-hydroxymethyl]-1,1-dimethyl-2-oxo-3-pentenyl $N, N$ -}

diisopropylcarbamate $((Z)-15 b)$

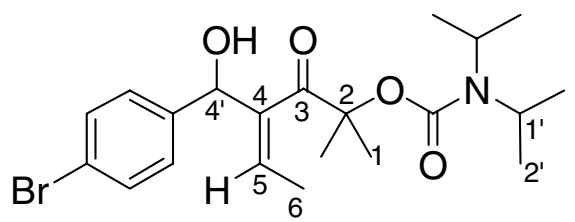

According to GEP 3, $150 \mathrm{mg}(0.58 \mathrm{mmol})$ of $8 \mathbf{b}$ resulted in $162 \mathrm{mg}(0.37 \mathrm{mmol}, 63 \%)(Z)-\mathbf{1 5 b}$, colourless solid; $\mathrm{R}_{\mathrm{f}}=$ $0.6\left(\mathrm{Et}_{2} \mathrm{O} /\right.$ pentane $\left.=1 / 2\right)$; m.p. $=129{ }^{\circ} \mathrm{C}$ (from the melt $) ;{ }^{1} \mathrm{H}-\mathrm{NMR}(300 \mathrm{MHz}): \delta=1.26\left(\mathrm{br} \mathrm{s},{ }^{3} J_{2}, 1_{1},=6.0 \mathrm{~Hz}, 12 \mathrm{H}, 2^{\prime}-\right.$ $\left.\mathrm{H}_{3}\right) ; 1.62,1.72\left(\mathrm{~s}, 6 \mathrm{H}, 1-\mathrm{H}_{3}\right) ; 1.65\left(\mathrm{dd},{ }^{3} J_{6,5}=1.1 \mathrm{~Hz}, 3 \mathrm{H}, 6-\mathrm{H}_{3}\right) ; 3.75-4.05\left(\mathrm{br} \mathrm{s}, 2 \mathrm{H}, 1\right.$ ' $\left.-\mathrm{H}_{1}\right) ; 4.42\left(\mathrm{~d},{ }^{3} J_{\mathrm{OH}, 4^{\prime}}=4.3 \mathrm{~Hz}\right.$, $1 \mathrm{H}, \mathrm{OH}) ; 5.19-5.27\left(\mathrm{dq},{ }^{3} J_{5,6}=1.1 \mathrm{~Hz}, 1 \mathrm{H}, 5-\mathrm{H}_{1}\right) ; 5.57\left(\mathrm{~d},{ }^{3} \mathrm{~J}_{4}\right.$, $\left.\mathrm{OH}=4.3 \mathrm{~Hz}, 1 \mathrm{H}, 4^{\prime}-\mathrm{H}_{1}\right) ; 7.20-7.29(\mathrm{~m}, 2 \mathrm{H}, \mathrm{Ph}) ; 7.43-$ 7.48 (m, $2 \mathrm{H}, \mathrm{Ph}) ;{ }^{13} \mathrm{C}-\mathrm{NMR}$ (75 MHz): $\delta=15.71$ (C-6); 20.62, $21.41(\mathrm{C}-2$ '); 25.38, $25.87(\mathrm{C}-1)$; 46.18, $47.03(\mathrm{C}-1$ '); 73.84 (C-4'); 83.69 (C-2); 121.13 (C ipso p-Br-Ph); 128.71, (C-5); 128.85, 131.14 (CH-Ph); 140.84, 145.90 (C-4, C ipso $\mathrm{Ph}) ; 154.61(\mathrm{NC}=\mathrm{O}) ; 207.99(\mathrm{C}-3) ; \mathrm{IR}(\mathrm{KBr}): \tilde{v}\left[\mathrm{~cm}^{-1}\right]=3478(\mathrm{O}-\mathrm{H}) ; 2998,2931(\mathrm{C}-\mathrm{H}) ; 1701(\mathrm{NC}=\mathrm{O}) ; 1666$ $(\mathrm{C}=\mathrm{C}-\mathrm{C}=\mathrm{O}) ; \quad$ ESI-MS: $\quad \mathrm{m} / \mathrm{z}=440.3,442.2 \quad[(\mathrm{M})] ; \quad 462.3, \quad 464.3 \quad\left[\left(\mathrm{M}+\mathrm{Na}^{+}\right)\right] ; \quad 478.3, \quad 480.3 \quad\left[\left(\mathrm{M}+\mathrm{K}^{+}\right)\right]$; $\mathrm{C}_{21} \mathrm{H}_{30} \mathrm{BrNO}_{4}(439.14)$ Calcd. C 57.28, H 6.87, N 3.18; Found C 57.34, H 6.80, N 3.09.

\section{(5E)-3-[(Z)-Ethylidene]-4-hydroxy-1,1,5-trimethyl-2-oxo-6-phenylhex-5-enyl $N, N$ - diisopropylcarbamate $((Z)-15 c)$}

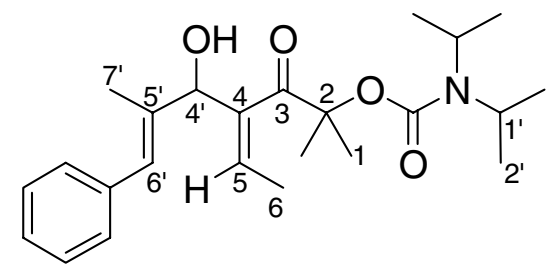

According to GEP 3, $149 \mathrm{mg}(0.58 \mathrm{mmol})$ of $\mathbf{8 b}$ resulted in $140 \mathrm{mg}(0.35 \mathrm{mmol}, 60 \%)(Z)-\mathbf{1 5 c}$, colourless liquid; $\mathrm{R}_{\mathrm{f}}=$ $0.5\left(\mathrm{Et}_{2} \mathrm{O} /\right.$ pentane $\left.=2 / 5\right) ;{ }^{1} \mathrm{H}-\mathrm{NMR}(300 \mathrm{MHz}): \delta=1.24\left(\mathrm{br} \mathrm{s},{ }^{3} J_{2},{ }_{1},=6.0 \mathrm{~Hz}, 12 \mathrm{H}, 2{ }^{\prime}-\mathrm{H}_{3}\right) ; 1.65,1.71\left(\mathrm{~s}, 6 \mathrm{H}, 1-\mathrm{H}_{3}\right)$; $1.73,1.76\left(\mathrm{dd},{ }^{3} J_{6,5}=7.1 \mathrm{~Hz}, 3 \mathrm{H}, 6-\mathrm{H}_{3}\right) ; 1.85\left(\mathrm{~d},{ }^{4} J_{7^{\prime}, 6^{\prime}}=1.3 \mathrm{~Hz}, 3 \mathrm{H}, 7\right.$ ' $\left.-\mathrm{H}_{3}\right) ; 3.74-4.00\left(\right.$ br s, $\left.2 \mathrm{H}, 1^{\prime}-\mathrm{H}_{1}\right) ; 3.81$ (d, $\left.{ }^{3} J_{\mathrm{OH}, 4^{\prime}}=4.4 \mathrm{~Hz}, 1 \mathrm{H}, \mathrm{OH}\right) ; 5.04\left(\mathrm{~d},{ }^{3} J_{4^{\prime}, \mathrm{OH}}=4.4 \mathrm{~Hz}, 1 \mathrm{H}, 4^{\prime}-\mathrm{H}_{1}\right) ; 5.75\left(\mathrm{dq},{ }^{3} J_{5,6}=7.1 \mathrm{~Hz},{ }^{4} J_{5,4},=0.8 \mathrm{~Hz}, 1 \mathrm{H}, 5-\mathrm{H}_{1}\right)$; $6.68\left(\mathrm{q},{ }^{4} J_{6}, 7,=1.3 \mathrm{~Hz}, 1 \mathrm{H}, 6{ }^{\prime}-\mathrm{H}_{1}\right) ; 7.16-7.40$ (m, $\left.5 \mathrm{H}, \mathrm{Ph}\right) ;{ }^{13} \mathrm{C}-\mathrm{NMR}(75 \mathrm{MHz}): \delta=15.57,15.81$ (C-6, C-7'); 20.69 , 21.42 (C-2'); 25.39, 25.85 (C-1); 46.21 (C-1'); 76.63 (C-4'); 83.69 (C-2); 126.34, 126.67, 128.07, 128.38, 129.03, 137.56, 143.59 (CH-Ph, C-4, C-5', C-6’), 128.38 (C-5); 137.86 (C-4); 154.44 (NC=O); 207.91 (C-3); IR (Film): $\tilde{\boldsymbol{V}}$ $\left[\mathrm{cm}^{-1}\right]=3428(\mathrm{O}-\mathrm{H}) ; 2972,2936(\mathrm{C}-\mathrm{H}) ; 1694$ (broad with shoulder, NC=O, C=C-C=O); ESI-MS: m/z = 402.5 $\left[\left(\mathrm{M}+\mathrm{H}^{+}\right)\right] ; 424.4\left[\left(\mathrm{M}+\mathrm{Na}^{+}\right)\right] ; \mathrm{C}_{24} \mathrm{H}_{35} \mathrm{NO}_{4}(401.26)$ Calcd. C 71.79, H 8.79, N 3.49; Found C 71.39, H 9.02, N 3.39. 


\section{(E)-1,1,5,5-Tetramethyl-2-oxo-3-hexenyl $N, N$-diisopropylcarbamate $(E)-12$}

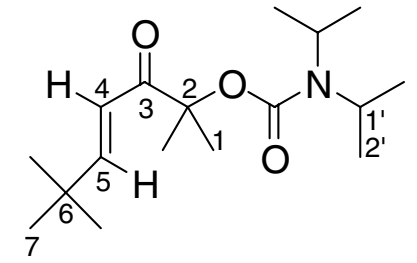

According to GEP 3, $187 \mathrm{mg}(0.62 \mathrm{mmol})$ of 8a resulted in $140 \mathrm{mg}(0.47 \mathrm{mmol}, 75 \%)(E)-\mathbf{1 2}$, as a colourless solid; $\mathrm{R}_{\mathrm{f}}$ $=0.3\left(\mathrm{Et}_{2} \mathrm{O} /\right.$ pentane $\left.=2 / 5\right) ;$ m.p. $=93{ }^{\circ} \mathrm{C}($ from the melt $) ;{ }^{1} \mathrm{H}-\mathrm{NMR}(300 \mathrm{MHz}): \delta=1.05\left(\mathrm{~s}, 9 \mathrm{H}, 7-\mathrm{H}_{3}\right) ; 1.13-1.38(\mathrm{br}$ m, $\left.12 \mathrm{H}, 2^{\prime}-\mathrm{H}_{3}\right) ; 1.49$ (s, $\left.6 \mathrm{H}, 1-\mathrm{H}_{3}\right) ; 3.79,3.92$ (br s, $\left.2 \mathrm{H}, 1^{\prime}-\mathrm{H}_{1}\right) ; 6.28\left(\mathrm{~d},{ }^{3} J_{4,5}=15.7 \mathrm{~Hz}, 1 \mathrm{H}, 4-\mathrm{H}_{1}\right) ; 6.98\left(\mathrm{~d},{ }^{3} J_{5,4}=\right.$ $\left.15.7 \mathrm{~Hz}, 1 \mathrm{H}, 5-\mathrm{H}_{1}\right) ;{ }^{13} \mathrm{C}-\mathrm{NMR}(75 \mathrm{MHz}): \delta=20.55,21.69$, (C-2'); 24.03 (C-1); 28.80 (C-7); 33.71 (C-6); $45.51,46.57$ (C-1'); 82.58 (C-2); 118.25 (C-4); 153.99 (NC=O); 156.91 (C-5); 198.75 (C-3); IR (KBr): $\tilde{v} \quad\left[\mathrm{~cm}^{-1}\right]=2962,2871(\mathrm{C}-$ $\mathrm{H}) ; 1704(\mathrm{NC}=\mathrm{O}) ; 1671,1634(\mathrm{C}=\mathrm{C}-\mathrm{C}=\mathrm{O})$; ESI-MS: $\mathrm{m} / \mathrm{z}=320.1\left[\left(\mathrm{M}+\mathrm{Na}^{+}\right)\right] ; \mathrm{C}_{17} \mathrm{H}_{31} \mathrm{NO}_{3}(297.23)$ Calcd. C 68.65, H 10.51, N 4.71; Found C 68.58, H 10.33, N 4.48.

\section{retro Aldol type reaction of $(Z)-10 a$}

$47 \mathrm{mg}(Z)-10 \mathrm{a}(0.12 \mathrm{mmol}, 1.0 \mathrm{eq})$ was dissolved in $4.0 \mathrm{~mL}$ THF and cooled to $-78^{\circ} \mathrm{C} .0 .07 \mathrm{~mL}$ of $1.6 \mathrm{M} n$ - $\mathrm{BuLi}$ in hexane $(0.12 \mathrm{mmol}, 1.0 \mathrm{eq})$ was added with stirring. After $20 \mathrm{~min}, 0.04 \mathrm{~mL}, 2$-methylbenzaldehyde $(0.34 \mathrm{mmol}, 3.0 \mathrm{eq})$ was added and the reaction mixture was stirred at room temperature for $3.5 \mathrm{~h}$. The reaction mixture was quenched with $3.0 \mathrm{~mL}$ of water and extracted with diethyl ether $(3 \times 20 \mathrm{~mL})$. The combined organic extracts were dried over anhydrous $\mathrm{MgSO}_{4}$ and then concentrated under vacuum. The crude material was purified by column chromatography (eluents: pentane/ $\left.\mathrm{Et}_{2} \mathrm{O}=5: 1\right)$ furnishing $14 \mathrm{mg}(E)-\mathbf{1 1 a}(0.03 \mathrm{mmol}, 30 \%), 18 \mathrm{mg}(E)$-11e $(0.04 \mathrm{mmol}, 38 \%)$ and $4 \mathrm{mg}(E)$-12 $(0.01 \mathrm{mmol}, 10 \%)$. 